CERN-EP/98-126

10 August 1998

\title{
Parameterization of $e$ and $\gamma$ initiated showers in the NOMAD lead-glass calorimeter
}

\author{
D. Autiero ${ }^{1}$ M. Baldo-Ceolin ${ }^{5}$ F. Bobisut ${ }^{5}$ P.W. Cattaneo ${ }^{6}$ V. Cavasinni $^{7}$ G. Collazuol ${ }^{5}$ \\ G. Conforto ${ }^{9}$ M. Contalbrigo ${ }^{5}$ C. Conta ${ }^{6}$ T. Del Prete ${ }^{7}$ A. De Santo $^{1}$ R. Ferrari ${ }^{6}$ \\ V. Flaminio ${ }^{7}$ M. Fraternali ${ }^{6}$ D. Gibin ${ }^{5}$ S.N. Gninenko ${ }^{4}$ G. Graziani ${ }^{3}$ A. Guglielmi ${ }^{5}$ \\ E. Iacopini ${ }^{3}$ S. Lacaprara ${ }^{5}$ A. Lupi ${ }^{3}$ M.M. Kirsanov ${ }^{4}$ A.V. Kovzelev ${ }^{4}$ L. La Rotonda ${ }^{2}$ \\ A. Lanza ${ }^{6}$ M. Laveder ${ }^{5}$ A. Marchionni ${ }^{3}$ F. Martelli ${ }^{9}$ M. Mezzetto ${ }^{5}$ D. Orestano ${ }^{8}$ \\ F. Pastore ${ }^{6}$ E. Pennacchio ${ }^{9}$ R. Petti ${ }^{10,6}$ G. Polesello ${ }^{6}$ G. Renzoni ${ }^{7}$ C. Roda ${ }^{7}$ \\ F. Salvatore ${ }^{6}$ A. Sconza ${ }^{5}$ A.N. Toropin ${ }^{4}$ M. Valdata-Nappi ${ }^{11,2}$ M. Veltri ${ }^{9}$ V. Vercesi ${ }^{6}$ \\ S.A. Volkov ${ }^{4}$
}

\begin{abstract}
A description of the response of the NOMAD electromagnetic calorimeter to electrons and photons is discussed. In particular the dependence of the two-dimensional shower shape on the angle and energy of the incident particle is parametrized by analytical functions. Some applications to the neutrino event reconstruction are also reported.
\end{abstract}

(Submitted to Nuclear Instruments and Methods in Physics Research A ) 


\footnotetext{
${ }^{1}$ CERN 1211 Geveve 23, Switzerland

${ }^{2}$ Dipartimento di Fisica, Università della Calabria and INFN, Gruppo collegato di Cosenza, Italy ${ }^{3}$ Dipartimento di Fisica, Università di Firenze and INFN, Sezione di Firenze, Italy

${ }^{4}$ Institute for Nuclear Research, INR, Moscow,Russia

${ }^{5}$ Dipartimento di Fisica, Università di Padova and INFN, Sezione di Padova, Italy

${ }^{6}$ Dipartimento di Fisica Nucleare e Teorica, Università di Pavia and INFN, Sezione di Pavia, Italy

${ }^{7}$ Dipartimento di Fisica, Università di Pisa and INFN, Sezione di Pisa, Italy

${ }^{8}$ Dipartimento di Fisica, Università di Roma Tre and INFN, Sezione di Roma III, Italy

${ }^{9}$ Dipartimento di Fisica, Università di Urbino and INFN, Sezione di Firenze, Italy

${ }^{10} \mathrm{e}$-mail: petti@pv.infn.it

${ }^{11}$ Now at Università di Perugia, Italy
} 


\section{Introduction}

The main goal of the WA96 experiment (NOMAD) is to search for the appearance of $\tau$ neutrinos in the CERN SPS wide band neutrino beam [1]. The $\nu_{\tau}$ 's are observed through their charged current interactions producing $\tau$ 's. These in turn are measured in the leptonic decay channels $e \bar{\nu}_{e} \nu_{\tau}, \mu \bar{\nu}_{\mu} \nu_{\tau}$ and in the hadronic channels $\pi^{-} \nu_{\tau}, \rho^{-} \nu_{\tau}$ and $\pi^{+} \pi^{-} \pi^{-} \nu_{\tau}$.

To distinguish $\tau$ decays from $\nu_{\mu}$ and $\nu_{e}$ charged and neutral current interactions NOMAD strongly relies on a technique which makes use of a series of cuts on kinematical variables, based on the momentum balance of the events.

In order to be sensitive to a large fraction of $\tau$ decay modes and to minimize the background contamination the NOMAD detector has been designed to:

- measure the charged tracks in the drift chamber target with high precision;

— identify and measure electrons and $\gamma$;

— identify and measure muons;

- achieve a high level of rejection against tracks faking electrons and muons.

A detailed description of the NOMAD detector and its performance has been given elsewhere [2]. In particular, the choice of a lead-glass calorimeter having a good intrinsic resolution, was found to be adequate to provide the required accuracy in measuring electron and $\gamma$ energies. The combined information coming from the calorimeter and a preshower detector located in front improves the $e$ identification and the separation of particles overlapping in the calorimeter.

The electromagnetic calorimeter of NOMAD consists of a matrix of 35 rows and 25 columns of lead-glass blocks (TF1-000), 19 radiation lengths deep $(\sim 500 \mathrm{~mm})$ and with a rectangular cross section of $79 \times 112 \mathrm{~mm}^{2}$. The Cerenkov light produced within the leadglass is detected by two stage photomultipliers (tetrodes) designed to operate in intense magnetic fields and placed at $45^{0}$ with respect to both the block axis and the magnetic field direction. The design, the performances and the calorimeter response to different particles are described in references [3] [4].

The particles produced in neutrino interactions in NOMAD have broad distributions both for the energy and for the impact angles on the calorimeter due to the kinematical conditions and to the presence of a magnetic field of $0.4 T$ [1] [2]. On the other hand, since the electromagnetic showers generated in the lead-glass are rather compact a strong dependence of the measured profile on the impact angle and on the energy of the particles is expected.

A detailed $e$ and $\gamma$ shower parametrization taking into account such dependences can provide a very accurate estimate of particle energy and position. In fact a three-dimensional description of the local behaviour of the shower (differential approach) includes the angular dependence and is very efficient for fast Monte Carlo (MC) simulations. Unfortunately the method is not compatible with a cluster reconstruction algorithm since the integration over the block dimensions introduces a further approximation and increases the time required for calculations. To this purpose, a model that can directly estimate the integral response as a function of the incidence angle and the energy of the particle should be chosen.

In the following an integral approach and its application to particle identification will be described. Section 2 provides details on the experimental and simulated samples used 
throughout. After a short discussion of the local properties of the showers inside the leadglass blocks of the NOMAD calorimeter (section 3), a full parameterization of the integral response, based on both test-beam measurements and $\mathrm{MC}$ simulations, is then presented in section 4. A quantitative check of the model reliability is discussed in the section 5 . Finally, the algorithm is applied to the reconstruction of the neutrino interactions inside the NOMAD detector (section 6). All the results presented in the following come from MC simulation unless otherwise declared.

\section{Data sample}

\section{$2.1 \quad$ Test-beam data}

Extensive measurements were performed with electron beams at the CERN PS and SPS using a lead-glass matrix of $16 \times 4$ blocks equipped with the same readout electronic chain as in the NOMAD experiment [3]. The module was placed on a movable platform in order to perform horizontal and vertical scans with the beam. In addition it was also rotated around two orthogonal axes to change the impact angles. A region covering about $2 \times 2$ lead-glass blocks was scanned uniformly at different impact angles ranging from $0^{0}$ to to $20^{0}$ to fully measure shower shapes at two different energies $E=5 \mathrm{GeV}$ and $E=10 \mathrm{GeV}$. A detailed description of the experimental set up can be found in reference [4].

The particle coordinates in the transverse plane were measured by means of two delay wire chambers (DWC) with $200 \mu \mathrm{m}$ resolution and the beam divergence was kept below 10 mrad. The estimated momentum bite was $\Delta p / p \sim 1 \%$. High statistics samples were taken throughout in order to reduce the statistical uncertainty and to have a reliable estimate of the intrinsic fluctuations in the energy deposition.

The overall alignment between the DWCs and the lead-glass module was calculated from the measured response to electrons impinging normally on the boundaries between two adjacent blocks. The corresponding systematic uncertainty in the impact position at the lead-glass front face was estimated to be less than $0.5 \mathrm{~mm}$.

Special runs were taken to simulate the NOMAD experimental conditions: the module was placed inside a magnet, providing a field with the same intensity and direction as inside NOMAD, and a prototype of the NOMAD preshower detector was also placed in front of the calorimeter.

\subsection{Monte Carlo simulation}

A full GEANT (version 3.15) simulation [5] of the calorimeter was performed, taking into account the Cerenkov light propagation within the lead-glass blocks. This was intended both to have access to configurations not directly available in the real data and to understand the local behaviour of the electromagnetic shower inside the lead-glass.

High statistics electron samples ( $\geq 50 \mathrm{k}$ events) were generated at $E_{0}=5 \mathrm{GeV}$ for impact angles ranging from $0^{0}$ to $25^{0}$ in one degree steps. The particle coordinates at the calorimeter front face were uniformly distributed within a $2 \times 2$ block matrix. The energy dependence at different angles was also investigated in the range $0.1 \div 80 \mathrm{GeV}$. In order to optimize the computation time, the number of events generated at each energy was fixed proportional to the fluctuations expected from the nominal energy resolution [4]. The comparison between test-beam results and MC predictions is discussed in detail in section 5.1. 


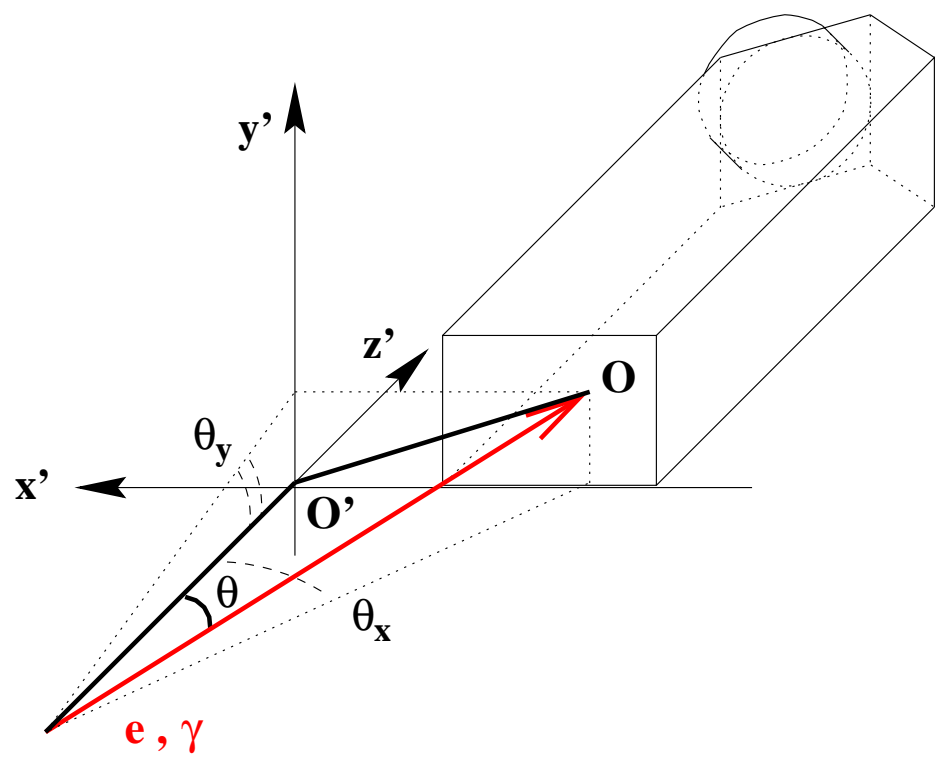

Figure 1: General reference frame.

The effects of the NOMAD magnetic field and preshower on shower shapes were individually studied with MC, in particular in the low energy region and for large impact angles. The presence of the lead converter in front of the calorimeter was found particularly relevant for photon initiated showers as expected. Actually a MC simulation was the only method to obtain a clean $\gamma$ sample at different impact angles and energy. The results are described in section 4.3. The magnetic field could, in principle, affect the measured response by changing the size of the sensitive area of the tetrode photocatode [3]. It was verified with special Monte Carlo simulations that the shower shapes were not sensitive to this effect, as expected, due to the large number of the particles in the shower which randomize the light collection at the photocatode.

\section{Shower development}

A brief description of the shower behaviour inside the NOMAD lead-glass counters $\left(X_{0} \sim 2.5\right.$ $\mathrm{cm})$ is presented in this section. Although most general features of the local response can be found in literature, the description given here is useful to understand the actual response to electromagnetic particles of the NOMAD calorimeter and to introduce the integral approach.

In the following a cylindrical coordinate system will be adopted throughout. The global reference frame (figure 1) is such that the calorimeter front face lies in the $X Y$ plane, the longest block size being along $X$ axis and the origin being at the impact point.

If no other specification is given, the transverse $X(Y)$ profile is defined as the local density of the signal as a function of $X(Y)$ coordinate, integrated over the longitudinal direction $z$. Similarly, the longitudinal profile will describe the shower development along $z$ integrated over the transverse plane. 


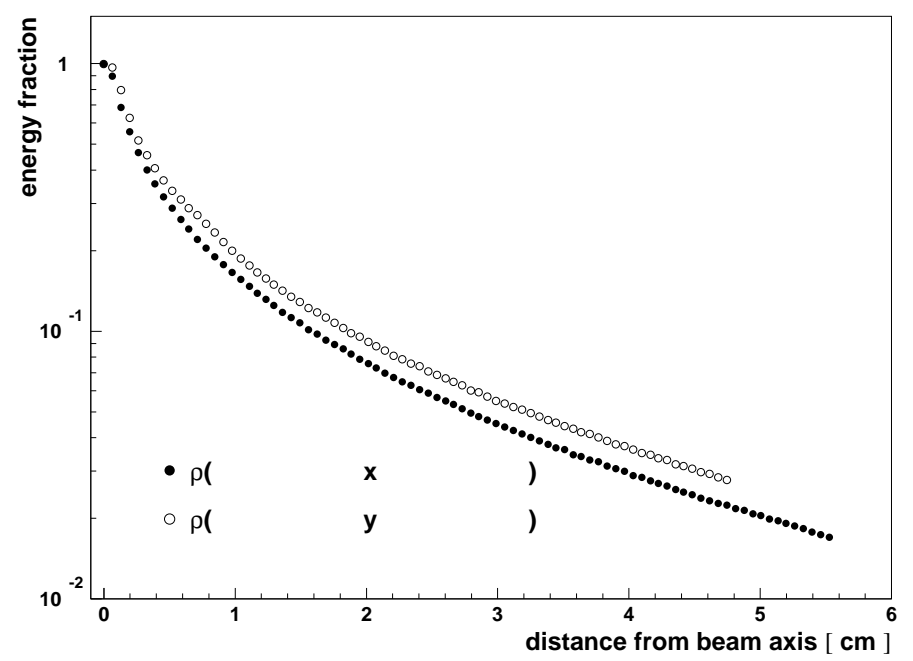

Figure 2: Transverse profile $\rho$ for $E_{0}=5 \mathrm{GeV}$. The two curves are normalized to 1 at $r=0$.
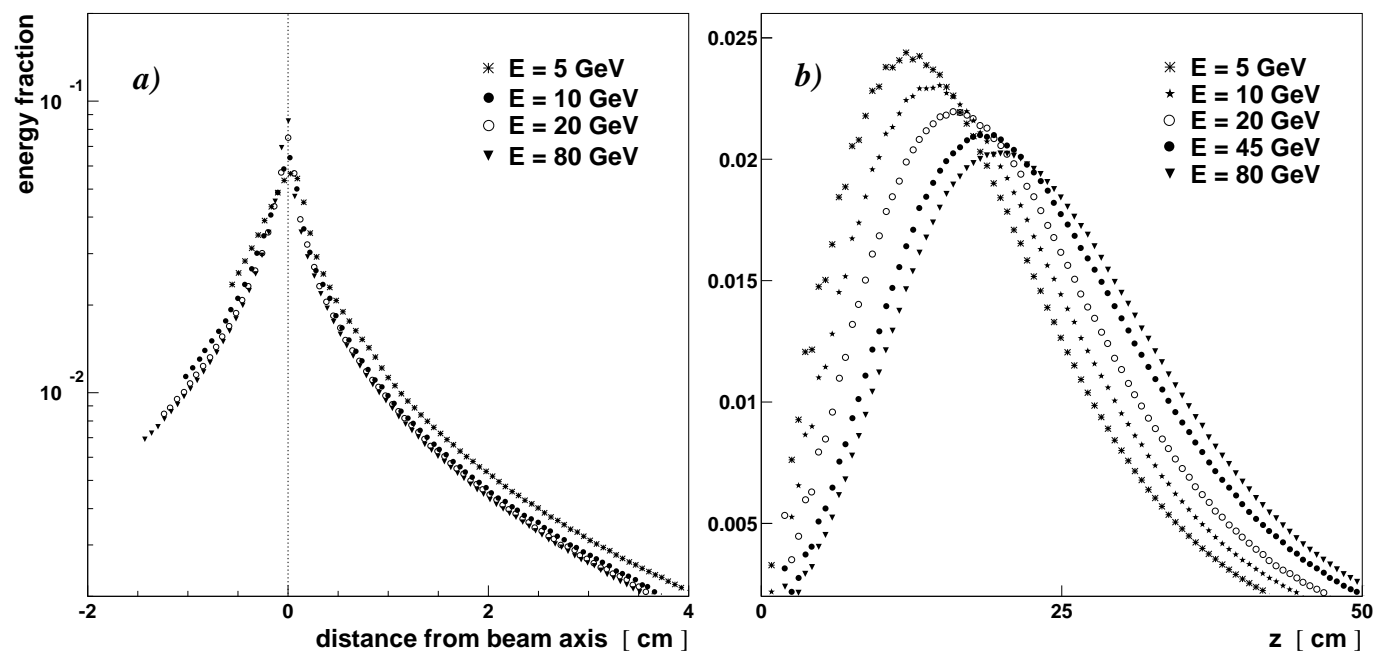

Figure 3: Differential profiles at different energies: a) transverse profile $\rho(y)$ and b) longitudinal profile $\xi(z)$. All curves are normalized to unit area. 


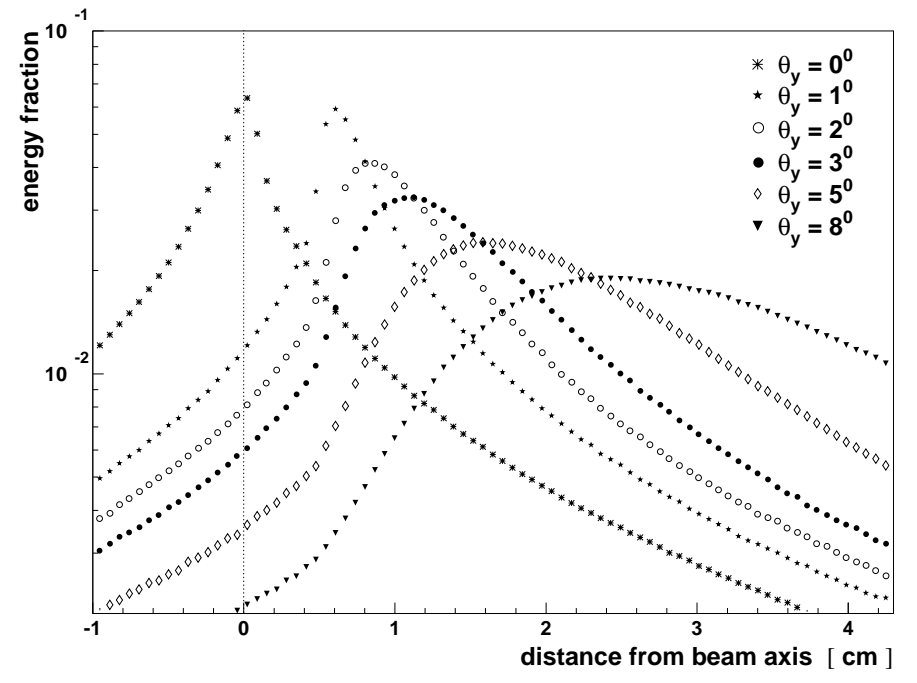

Figure 4: Angular dependence of the transverse profile $\rho(y)$ for $E=10 \mathrm{GeV}$. All curves are normalized to unit area.

\subsection{Differential profiles}

For a particle impinging normally to the $X Y$ plane, the transverse profile $\rho(x, y)$ is characterized by an exponential decay [6] [7] [8]:

$$
\rho(x, y)=\sum_{i} a_{i} e^{-r / b_{i}} \quad r=\sqrt{x^{2}+y^{2}}
$$

where the sum extends to all the shower components (in the following $i=1,3$ will be taken), $a_{i}$ is the weight of each component and $b_{i}$ its characteristic attenuation length. The main contribution arises from the first term which describes the sharply collimated central core of the shower while the remaining terms (which sometimes are neglected as an acceptable approximation for pattern recognition) take into account the long tail of the shower.

The asymmetry in the block dimensions of the NOMAD calorimeter introduces differences in the light collection efficiency along the two coordinates inside the block. This fact modifies the physical distribution $\rho(x, y)$ which has no longer a cylindrical symmetry, as shown in figure 2.

The longitudinal development of the electromagnetic cascade can be parametrized by the distribution [9]:

$$
\xi(z)=A z^{\alpha} e^{-z / \lambda}
$$

where $A$ is a normalization factor, $\lambda$ is a parameter which varies very slowly with the energy of the incident particle $E$ and $\alpha$ is a linear function of $\log (E)$.

\subsection{Angular effects and energy dependence}

The transverse differential profile $\rho(x, y)$, integrated along the longitudinal direction $z$, has a weak dependence on the energy of the particle at $\theta=0^{0}$ (figure 3a). Actually, on the average, 
only about $10 \%$ of the energy lies outside a cylinder with a Molière radius $R_{M}\left(R_{M} \sim 4\right.$ $\mathrm{cm}$ in NOMAD).

On the other hand, the longitudinal distribution $\xi(z)$ is rather sensitive to the energy of the particle, resulting in a shift of the peak for the observed energy distribution (figure $3 \mathrm{~b}$ ) which generates a variation in the position of the longitudinal centre of gravity of the shower. This energy dependence can be described by the well known logarithmic shape [9] [10].

When the impact angle $\theta$ is different from $0^{0}$ the longitudinal and radial shapes get mixed together. In such a situation an asymmetric shape of the transverse profile is then clearly visible and produces a shift in the position of the peak (figure 4). As a consequence, the longitudinal component induces a strong energy dependence also in the radial profile $\rho(x, y)^{1}$.

\section{Integral response}

To evaluate the average response of the calorimeter it is necessary to know the integral response of a lead-glass block:

$$
F(x, y, \theta, \phi, E)=N \int_{x-\Delta x_{0} / 2}^{x+\Delta x_{0} / 2} d x^{\prime} \int_{y-\Delta y_{0} / 2}^{y+\Delta y_{0} / 2} d y^{\prime} \rho\left(x^{\prime}, y^{\prime}, E\right)
$$

that is the integral over the block of the differential profile $\rho$. The overall normalization factor $N$ is defined by the total energy released in the calorimeter by the particle:

$$
1 / N=\sum_{k=-\infty}^{+\infty} \sum_{m=-\infty}^{+\infty} F\left(x+k \Delta x_{0}, y+m \Delta y_{0}, \theta, \phi, E\right)
$$

From now on the normalization factor will be omitted.

The integral response $F(x, y, \theta, \phi, E)$ is an estimate of the average energy fraction released in the block itself.

\subsection{Response parameterization}

Even in the approximation of a cylindrical symmetry for $\rho(x, y, E)$, the integral response cannot be easily factorized in single one-dimensional projections due to the finite integrals appearing in equation (3). Moreover the asymmetry in the block sizes introduces a further effect in the distribution itself in addition to the simple geometrical one as previously discussed in equation (3).

Nevertheless, under the hypothesis of small lateral leakage (when the energy released at a distance $d \geq \sqrt{\left(\Delta x_{0}\right)^{2}+\left(\Delta y_{0}\right)^{2}}$ from the impact point is negligible), it is still possible to obtain a good approximation by extending the domain of integration along the projecting direction:

$$
F(x, y, \theta, \phi, E) \sim F_{x}(x, \theta, \phi, E) \times F_{y}(y, \theta, \phi, E)
$$

\footnotetext{
${ }^{1}$ In the following the energy dependence will be explicitely written as $\rho(x, y, E)$, while the angular dependence will be considered as part of the rotation of the reference frame.
} 

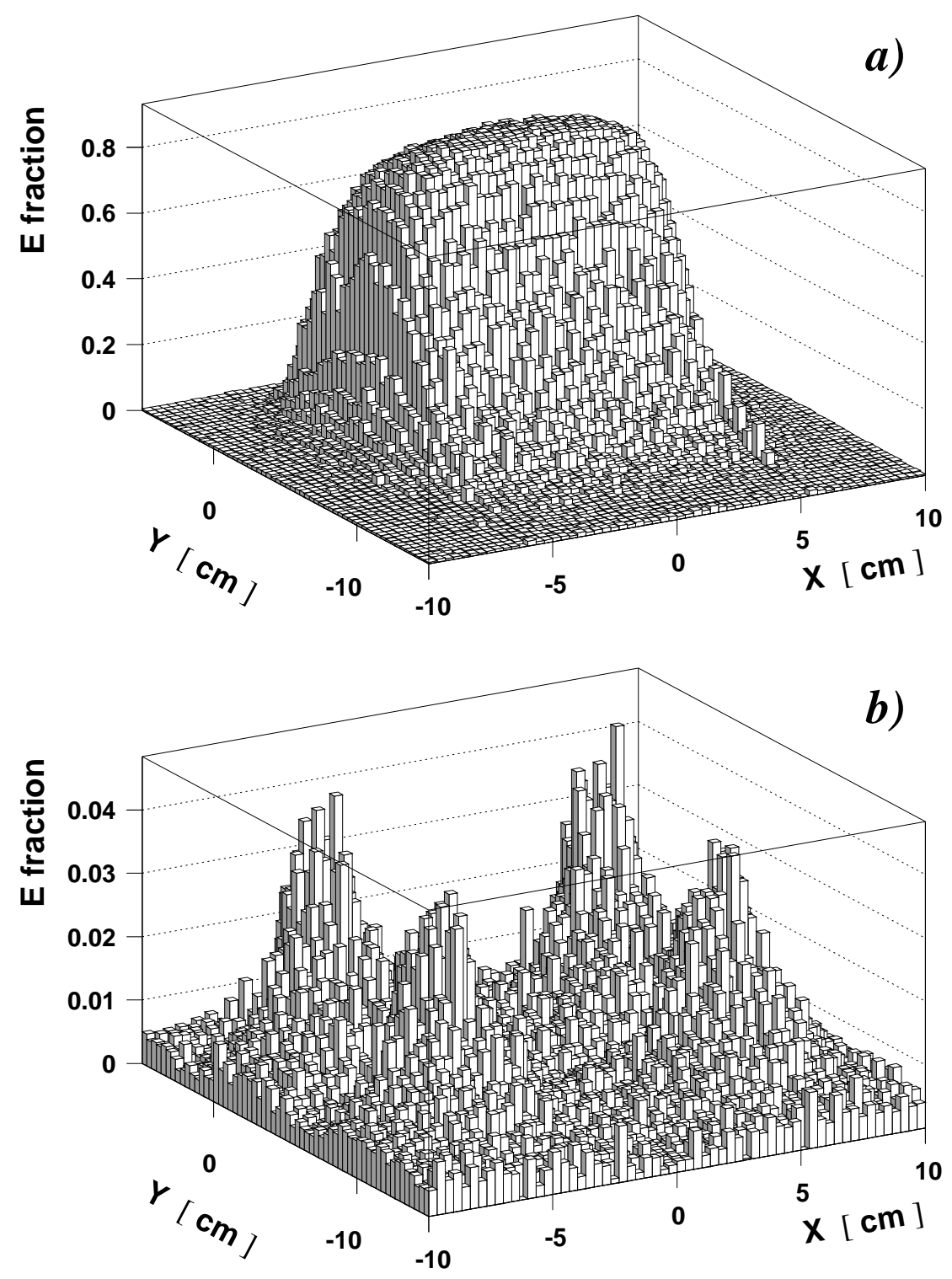

Figure 5: a) Integral shower profile $F\left(x, y, \theta_{0}=10^{0}, \phi_{0}=90^{\circ}, E_{0}=10 \mathrm{GeV}\right)$; b) Error on factorization $\left|F\left(x, y, \theta_{0}, \phi_{0}, E_{0}\right)-F_{x}\left(x, \theta_{0}, \phi_{0}, E_{0}\right) \times F_{y}\left(y, \theta_{0}, \phi_{0}, E_{0}\right)\right|$. 


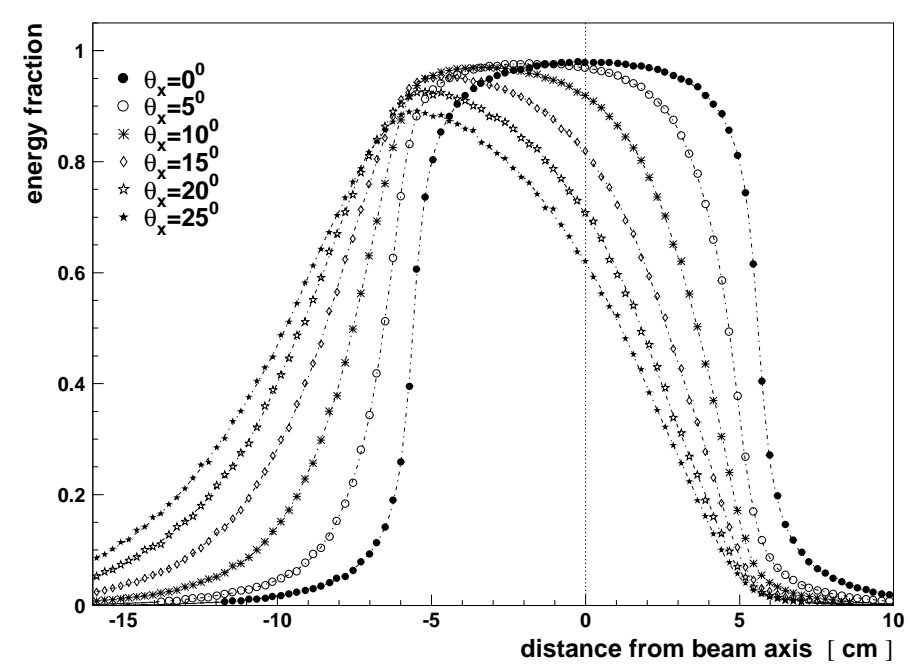

Figure 6: Angular dependence of the integral shower profile $F_{x}\left(x, \theta_{x}, E_{0}=5 \mathrm{GeV}\right)$ : the fitted curves are the corresponding functions parametrized according to equation (10).

where

$$
F_{x}(x, \theta, \phi, E)=\int_{x-\Delta x_{0} / 2}^{x+\Delta x_{0} / 2} d x^{\prime} \int_{-\infty}^{+\infty} d y^{\prime} \rho\left(x^{\prime}, y^{\prime}, E\right)
$$

A similar expression can be written for $F_{y}(y, \theta, \phi, E)$.

The condition of small lateral leakage is met in the NOMAD calorimeter [4] and the integral response expected for a single block can be factorized with good approximation by the product of the corresponding row and column. Figures 5a-b show the actual integral profile $F(x, y, \theta, \phi, E)$ and its relative difference from the factorized profile for electrons in a particular kinematical configuration $\left(\theta_{0}=10^{\circ}, \phi_{0}=90^{\circ}\right)$ : the small differences observed in figure $5 \mathrm{~b}(\leq 4 \%)$ support the factorization hypotesis.

For an incidence angle $\theta=0^{0}$, an analytical expression of $F_{x}$ (or equivalently $F_{y}$ ) can be obtained from equations (1) and (6) assuming, as a first approximation, a perfect cylindrical symmetry:

$$
F_{x}(x, \theta=0, E)=\sum_{i} a_{i} \int_{x-\Delta x_{0} / 2}^{x+\Delta x_{0} / 2} d x^{\prime} \int_{-\infty}^{+\infty} d y^{\prime} \exp \left[-\sqrt{\left(x^{\prime}\right)^{2}+\left(y^{\prime}\right)^{2}} / b_{i}\right]
$$

This equation can be integrated numerically with the Kelvin function $K_{1}$ [11]:

$$
F_{x}(x, \theta=0, E)=2 \sum_{i} a_{i} \int_{x-\Delta x_{0} / 2}^{x+\Delta x_{0} / 2} d x^{\prime} x^{\prime} K_{1}\left(\left|x^{\prime}\right| / b_{i}\right)
$$

The above analytical solution can be approximated by the expression:

$$
F_{x}(x, \theta=0, E)=\frac{1}{\pi} \sum_{i} a_{i} \times \arctan \left(x / b_{i}\right)
$$

Equation (9) provides a phenomenological function which can be directly superimposed to the experimental data even when the differential profile $\rho(x, y, E)$ has no symmetry. 

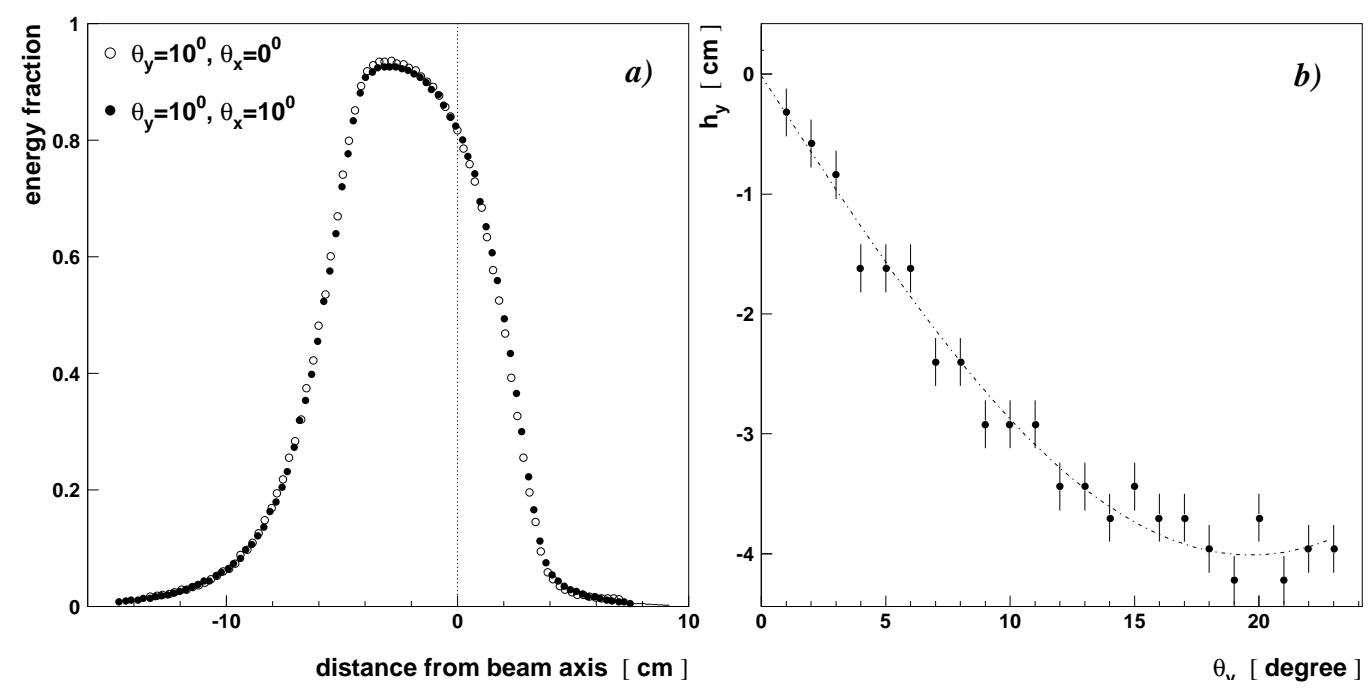

Figure 7: a) Example of the effect of $\theta_{x}$ on the integral profile $F_{y}\left(y, \theta_{y}, E_{0}=5 \mathrm{GeV}\right) \theta_{y}=10^{0}$ and b) systematic shift in the position of maximum energy deposition $h_{y}\left(\theta_{y}, E=E_{0}\right)$.

In order to take into account the contribution from the longitudinal profile for $\theta \neq 0^{0}$, a new term is added in the expression (9):

$$
\begin{aligned}
F_{x}(x, \theta, \phi, E) & =\frac{1}{\pi} \sum_{i} a_{i} \times \arctan \left(x / b_{i}\right)+ \\
& +\sum_{j} \frac{1}{c_{j} \sqrt{2 \pi}} \exp \left[-\left(x-d_{j}\right)^{2} / 2 c_{j}^{2}\right]
\end{aligned}
$$

where $a_{i}, b_{i}, c_{j}, d_{j}$ are parameters depending on the kinematical variables. The analytical expression of the second term of equation 10 is due to the fact that the longitudinal profile $\xi(z)$ for $\theta=0^{0}$ can also be approximated by a superposition of gaussians. The above assumption results in a linear combination of the transverse and longitudinal contributions which are considered as independent in this approximation.

\subsubsection{Angular factorization}

As expected, the integral response is very sensitive to the impact angle. Actually, the angular dependence increases the transverse radius of the shower which is then shared among several blocks along the direction of the incoming particle. The shower profiles begin to show an asymmetric shape and a systematic shift in the position of the maximum (figure 6). A shower profile analysis based on $F(x, y, \theta, \phi, E)$ can correctly describe the energy distribution between the cells of a cluster when a global normalization factor is provided ${ }^{2}$ (equation (4)).

\footnotetext{
${ }^{2}$ Since the sum of the $F(x, y, \theta, \phi, E)$ function over the full calorimeter is fixed to one by definition, this approach is not sensitive to angular effects on the total measured energy. Actually, a small dependence is observed, mainly related to a variation of the light collection efficiency with the impact angles inside the lead-glass counters. This correction should then be taken into account for the final energy reconstruction [4].
} 


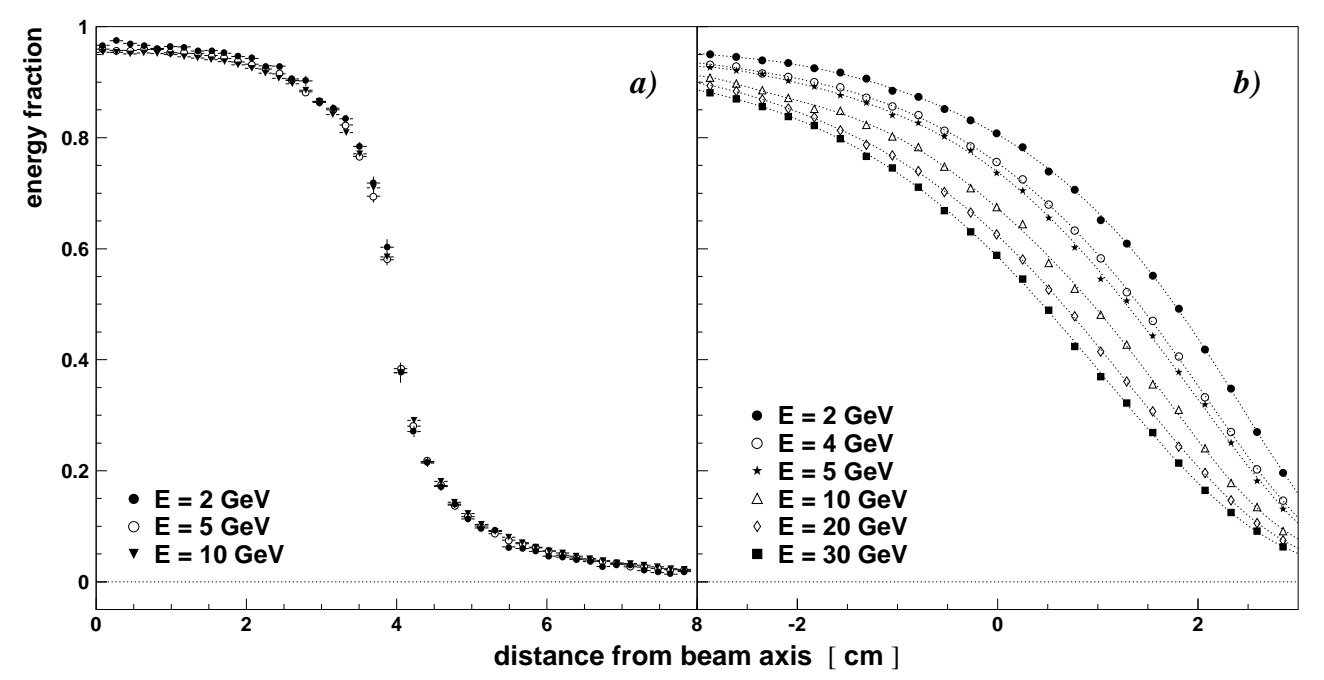

Figure 8: Integral shower profile $F_{y}\left(y, \theta_{y}, E\right)$ for a) $\theta_{y}=0^{0}$ and $\left.b\right) \theta_{y}=10^{0}$. The dotted lines are fitted according to equation (10).

In general the impact angle of a track can be described as the effect of a simple rotation of the reference frame and, in turn, of the domain of integration. This rotation is also the product of two different rotations along each of the two cartesian axis (X and $\mathrm{Y}$ ). It is thus possible to operate a full factorization of the angular dependence in the integral profiles using the angles $\theta_{x}$ and $\theta_{y}$ defined as:

$$
\left\{\begin{array}{l}
\tan \theta_{x}=\tan \theta \times \cos \phi \\
\tan \theta_{y}=\tan \theta \times \sin \phi
\end{array}\right.
$$

The integral profiles along each of the two axis depend only on the corresponding angle. Figure 7a shows this behaviour for electrons of $5 \mathrm{GeV}$ energy and $\theta_{y}=10^{0}$ : the $F_{y}$ function does not depend on the $\theta_{x}$ angle (the same is true for $F_{x}$ and $\theta_{y}$ ).

Assuming that the transverse systematic shift $h(\theta, E)$ is essentially related to the corresponding centre of gravity coordinates inside the lead-glass block, it is possible to get such a shift using only simple geometrical considerations:

$$
\left\{\begin{array}{l}
h_{x}\left(\theta_{x}, E=E_{0}\right)=\frac{\Delta x_{0}}{2} \sin \left(k_{x} \theta_{x}\right) \\
h_{y}\left(\theta_{y}, E=E_{0}\right)=\frac{\Delta y_{0}}{2} \sin \left(k_{y} \theta_{y}\right)
\end{array}\right.
$$

where a fixed energy $E_{0}$ is assumed for simplicity and $k_{x}$ and $k_{y}$ are constant parameters. Equation (12) is in good agreement with the observed behaviour as shown in figure $7 \mathrm{~b}$. Due to the finite cell size, the maximum shift is achieved for half of the cell width. Since the observed shift is related to the position $D$ of the longitudinal centre of gravity, in the small-angle approximation such parameters are defined as: $k_{x} \times \Delta x_{0} / 2 \equiv k_{y} \times \Delta y_{0} / 2=D$. 

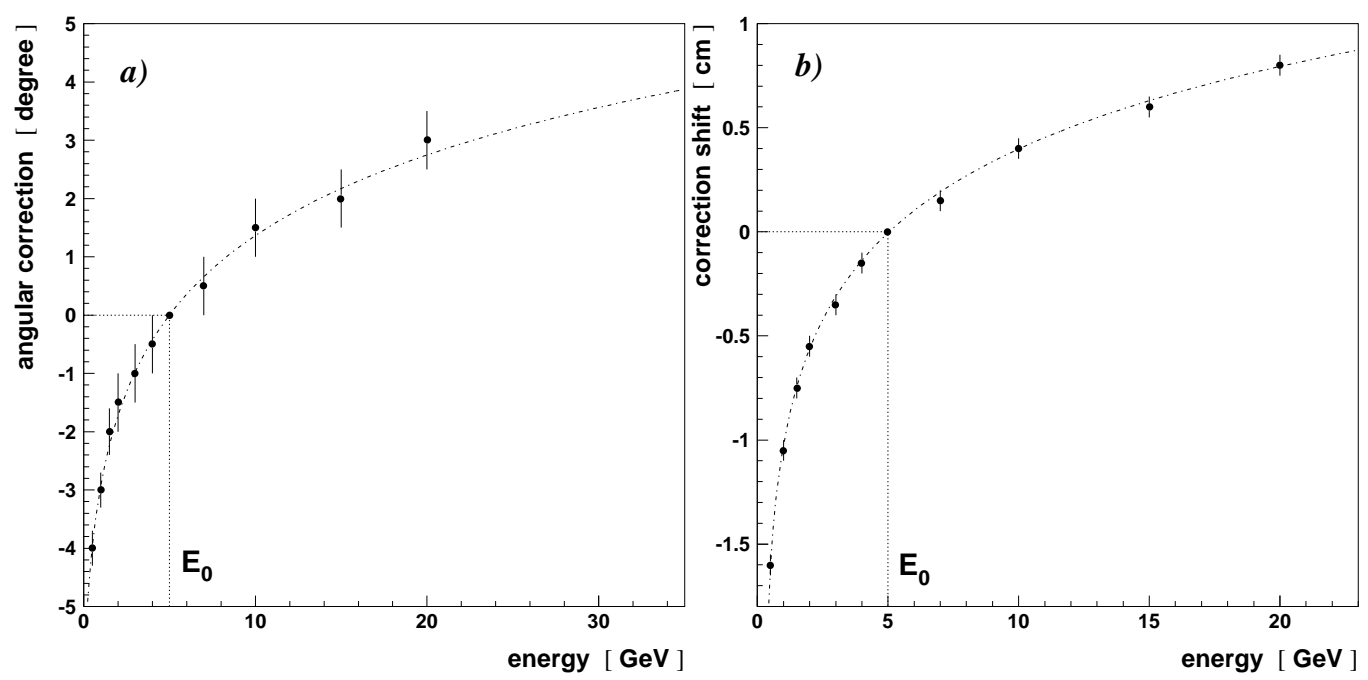

Figure 9: Energy scaling in shower shape parameterization: a) the angular correction $f\left(\theta_{y}, E, E_{0}=5 \mathrm{GeV}\right)$ at $\theta_{y}=15^{0}$ and b) the position shift $g_{2}\left(\theta_{x}, E, E_{0}=5 \mathrm{GeV}\right)$ at $\theta_{x}=20^{0}$ (see text).

\subsubsection{Energy scaling}

Since the integration is merely a geometrical step the integral distributions and the differential profiles (section 3.2) have the same behaviour: this means that a strong energy dependence can be measured only for $\theta \neq 0^{0}$ (figure $8 \mathrm{a}-\mathrm{b}$ ).

The same type of function can describe both the longitudinal profile $\xi(z)$, integrated along the radial size, and the longitudinal distributions of the energy released at a fixed radial distance. We can then imagine dividing the electromagnetic shower in several longitudinal slices and to assign to each of them its energy content. This energy will be higher and higher as the shower centre $(r=0)$ approaches and its longitudinal distribution will be described by the same function used for the global longitudinal profile.

Assuming that the energy dependence in the radial profile for $\theta \neq 0^{0}$ is due only to the contribution of the longitudinal component, it is possible to find a relationship between impact angle and visible energy. The number of slices which contribute to the radial profile (and to the energy as a consequence) at a fixed radial distance will increase with the impact angle $\theta$. The same configuration for the integral profile can then be associated to different values of the kinematical variables.

Due to the logarithmic dependence in the longitudinal component and to the linearity of the rotation, a logarithmic relationship $f\left(\theta, E, E_{0}\right)$ between the resulting 'effective' angles $\theta^{\prime}$ and the energy is expected. An example of this simple scaling law (for $\theta_{y}=15^{\circ}$ ) is shown in figure 9a, where the integral radial profile for $E_{0}=5 \mathrm{GeV}$ is assumed as a reference. For $E>E_{0}$ the visible energy will be higher: the same configuration can be obtained from the profile $E_{0}$ by simply increasing the effective angle $\theta^{\prime}$ in the reference profile (the angular corrections are positive). The reverse applies for $E<E_{0}$.

The coordinates in the integral distributions $F_{x}\left(x, \theta_{x}, E\right)$ and $F_{y}\left(y, \theta_{y}, E\right)$ are function of the actual kinematical parameters $\left(\theta_{x}, E\right)$ and $\left(\theta_{y}, E\right)$ at the impact point respectively. In 


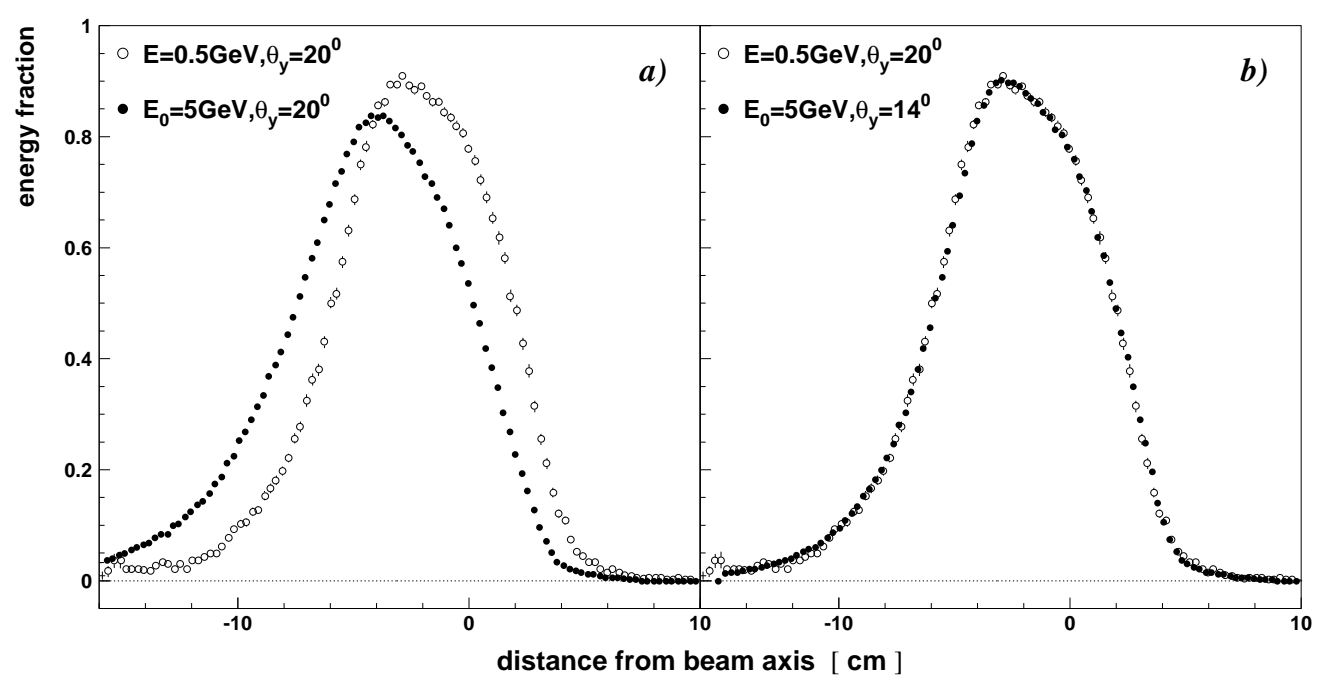

Figure 10: Global effect of the energy scaling law for $F_{y}\left(y, \theta_{y}, E\right)$ : a) integral shapes not corrected and b) energy corrections have been taken into account.

order to reintroduce such a dependence, the position of the reference profile should be rescaled taking into account the transverse shift in the maximum energy deposition (equation (12)). A correction shift $g_{1}\left(\theta^{\prime}, \theta, E_{0}\right)$ is thus applied at the fixed energy $E=E_{0}$. Moreover another correction $g_{2}\left(\theta, E, E_{0}\right)$ is needed to get the final position in the profile at the impact energy $E$, since the coordinate of the longitudinal centre of gravity is a logarithmic function of the particle energy $E$ (section 3.2). Figure $9 \mathrm{~b}$ shows this correction: its meaning is similar to that of the previous angular correction.

A general scaling law is obtained in this way and, as a consequence, the resulting parameterization can describe the integral profiles in terms of just one reference energy $E_{0}$ :

$$
\left\{\begin{array}{l}
F_{d}\left(d, \theta_{d}, E\right) \equiv F_{d}\left(d^{\prime}, \theta_{d}^{\prime}, E_{0}\right) \\
\theta_{d}^{\prime}=\theta_{d}+f\left(\theta_{d}, E, E_{0}\right) \\
d^{\prime}=d+g_{1}\left(\theta_{d}^{\prime}, \theta_{d}, E_{0}\right)+g_{2}\left(\theta_{d}, E, E_{0}\right) \quad d=x, y
\end{array}\right.
$$

where $g_{1}\left(\theta_{d}^{\prime}, \theta_{d}, E_{0}\right)=\left[h_{d}\left(\theta_{d}, E_{0}\right)-h_{d}\left(\theta_{d}^{\prime}, E_{0}\right)\right]$ and $f$ and $g_{2}$ are linear functions in $\log (E)$ for a fixed impact angle $\theta_{d}$ (figure $9 \mathrm{a}-\mathrm{b}$ ). The corresponding position of the maximum energy deposition is:

$$
h_{d}\left(\theta_{d}, E\right) \equiv h_{d}^{\prime}\left(\theta_{d}, E, E_{0}\right)=h_{d}\left(\theta_{d}, E_{0}\right)+g_{2}\left(\theta_{d}, E, E_{0}\right)
$$

The reference energy $E_{0}$, of course, can be fixed arbitrarily. The choice of $E_{0}=5 \mathrm{GeV}$ in figure $9 \mathrm{a}-\mathrm{b}$ is motivated by the need to optimize the parameterization in the lowest energy spectrum region, where the energy dependence becomes more important. On the other hand, this value is similar to the energies of the electrons expected from $\tau$ decay [1]. The effect of the full set of energy corrections on the integral distributions is reported in figure 10a-b. 

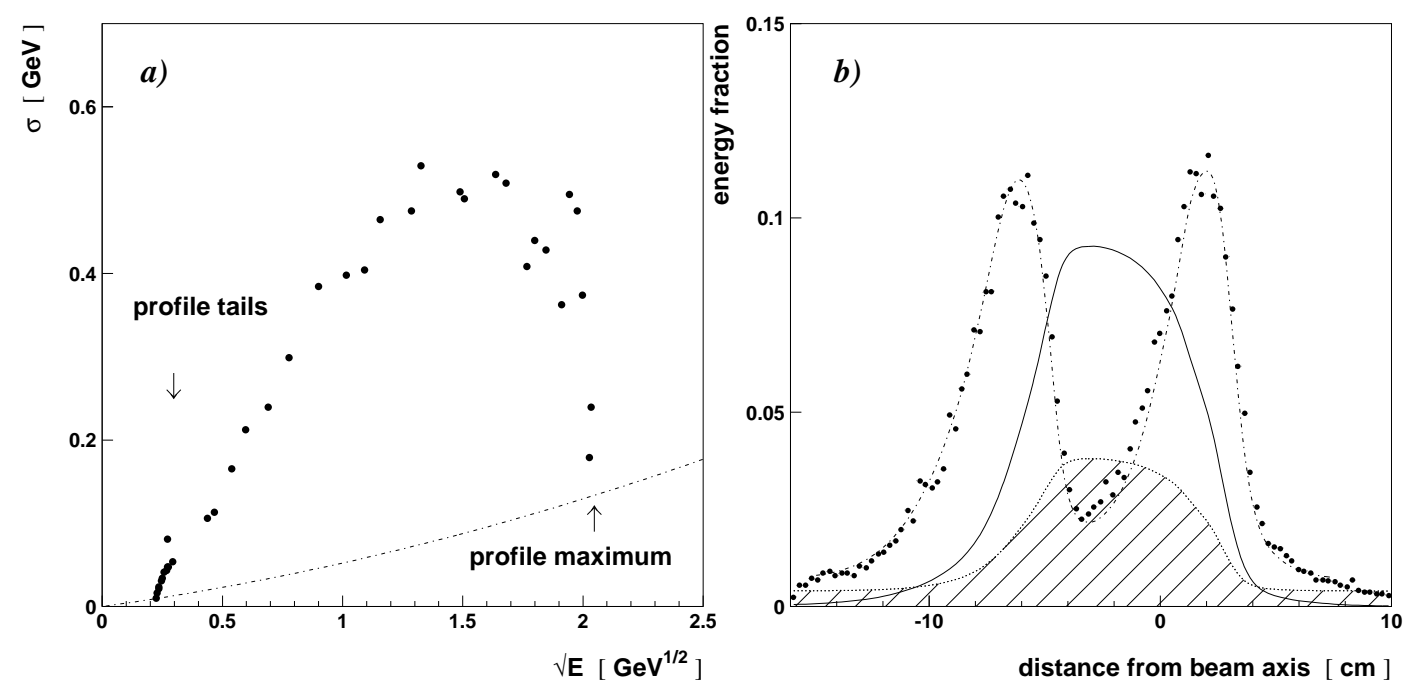

Figure 11: a) Fluctuations (rms) as a function of the square root of the average energy deposited in a row of the main nonet for electrons impinging at $\theta_{y}=10^{0}$ with a fixed energy $E_{0}=5 \mathrm{GeV}$. The dotted line is the predicted behaviour from the overall energy resolution of the calorimeter (see text). b) Shower fluctuations $\sigma_{y}\left(y, \theta_{y}, E_{0}=5 \mathrm{GeV}\right.$ ) at $\theta_{y}=10^{0}$ : the dotted line is the parametrized function and the hatched area is a calculation based on the calorimeter nominal resolution. The corresponding average shower profile $F_{y}\left(y, \theta_{y}, E_{0}\right)$ is also shown as solid line for comparison (multiplied by a factor 0.1).

\subsection{Shower fluctuations}

The knowledge of the fluctuations associated to the energy deposition is a crucial point in order to compare the predicted average shower profile $F$ with the actual energy measured in the calorimeter. For electromagnetic showers in lead-glass the overall shower fluctuations are small and mainly related to the photostatistics [4]. This is true for the total energy deposited by well contained particles. On the other hand, the local fluctuations induced in a single cell of the cluster are dominated by the charged tracks escaping the boundaries of the block itself. As a result of the incomplete containment of the shower, the energy deposition inside the cells fluctuates as a function of the distance between the impact point and the centre of the cells.

In figure 11a the relationship between the average and rms of the energy distribution measured in a single block is shown for $E=5 \mathrm{GeV}$ and $\theta_{y}=10^{0}$. The distance of the impact point from the block centre was changed in $1 \mathrm{~mm}$ step to fully explore the shower shape. The curve describing the calorimeter nominal energy resolution for a single cell [4], based on data collected at normal incidence, is well below the observed fluctuations except the point corresponding to the profile maximum (where by definition they coincide since most of the shower is contained in the block itself).

Due to the normalization condition (equation (4)) the integral functions $F, F_{x}$ and $F_{y}$ define only the relative energy deposition inside the cells of the cluster. The corresponding $\operatorname{rms} \sigma, \sigma_{x}$ and $\sigma_{y}$ are then equivalent to the fluctuations associated to the pure containment of the shower. This can be seen from figure 11b, where at the shower maximum such fluctuations are smaller than expected from the overall energy resolution. Actually, referring to the same 

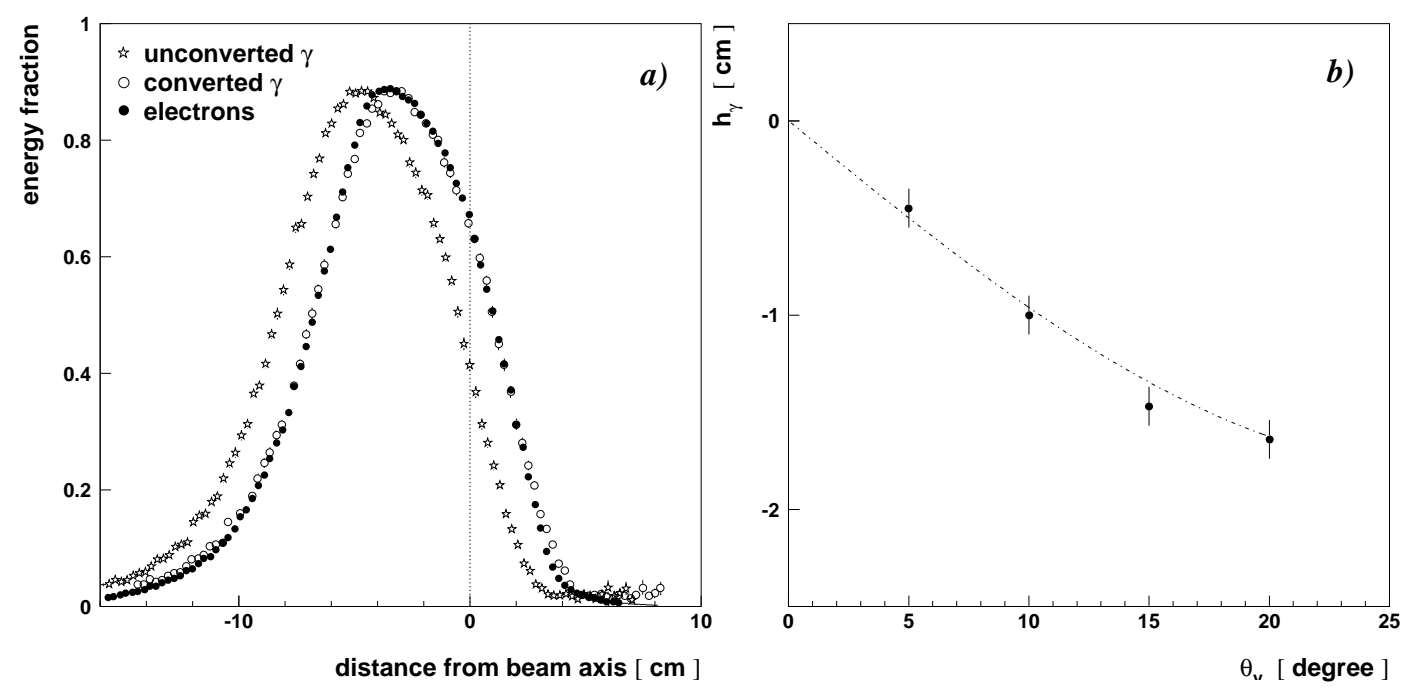

Figure 12: a) Comparison of $F_{y}\left(y, \theta_{y}=15^{0}, E_{0}=5 \mathrm{GeV}\right)$ shower shapes for converted $\gamma$, unconverted $\gamma$ and electrons. b) Position shift $h_{\gamma}\left(\theta_{y}, E=E_{0}\right)$ as a function of the impact angle $\theta_{y}$.

figure, $\sigma_{y}$ would be exactly zero for an infinite cell size because $F_{y} \equiv 1$ in this case. The additional fluctuations on the total energy measured in the calorimeter (i.e. photostatistics and energy deposition), which are cancelled out in the ratio to build $F$ and $\sigma$ functions, are reintroduced when the normalization of the shower profile calculation is performed ${ }^{3}$ (see also section 5).

Function $\sigma$ can then be related to the integral profile itself since this gives the best estimation of the containment factor:

$$
\sigma_{d}\left(d, \theta_{d}, E\right)=s(\sqrt{E}) \times \sigma_{d}^{\prime}\left(d, \theta_{d}, E\right) \quad d=x, y
$$

where $s(\sqrt{E})$ is a general scaling function depending only on the particle energy. The actual containement term $\sigma_{d}^{\prime}\left(d, \theta_{d}, E\right)$ is defined ${ }^{4}$ as:

$$
\sigma_{d}^{\prime}\left(d, \theta_{d}, E\right)=F_{d}\left(d, \theta_{d}, E\right) \times\left[1-F_{d}\left(d, \theta_{d}, E\right)\right] \oplus \alpha
$$

where $\alpha$ is a constant which takes into account systematic effects like calibration errors, digitization ${ }^{5}$ etc.

\footnotetext{
${ }^{3}$ In principle this normalization can be obtained by using even a single cell of the cluster. This is useful in order to limit overlap problems in case of close clusters and provides an acceptable estimation of the cluster energy. On the other hand, the procedure can bias the rms calculation since the influence of the containment factor on the estimated energy increases by reducing the number of cells considered. It is then important to maximize the actual energy deposit used to normalize the shower profile.

${ }^{4}$ Some deviations from equation (16) are observed when the particle energy is not fully contained in the considered nonet (e.g. for large angles). This is related to the definition of the adopted F functions and could be corrected by extending the shower calculation outside the main nonet.

${ }^{5}$ The best way to correct for digitization is to add a fixed term to the final normalized rms. Actually, the normalization process produce an energy-dependent $\sigma_{d}$ and the same is true for the $\alpha$ contribution. To compensate for this effect the constant term is introduced before the scaling function $s(\sqrt{E})$ is applied.
} 
The corresponding rms for the two-dimensional profile is defined as:

$$
\sigma\left(x, y, \theta_{x}, \theta_{y}, E\right) \equiv \sigma_{x}\left(x, \theta_{x}, E\right) \oplus \sigma_{y}\left(y, \theta_{y}, E\right)
$$

The expression (17) does not take into account the correlations between the projected shapes. As a consequence the resulting fluctuations are slightly overestimated and this fact partially compensates the error introduced on the factorization of the $F\left(x, y, \theta_{x}, \theta_{y}, E\right)$ profile.

\subsection{Photon initiated showers}

No difference between $e$ and $\gamma$ shower shapes is found in the case of photons which have converted in the preshower placed in front of the NOMAD calorimeter.

On the other hand, when the photon does not convert in the preshower, its electromagnetic shower will develop later inside the lead-glass because of the larger interaction length for photons $\left(X_{\gamma} \sim 9 / 7 X_{e}\right)$ and of the distance between the preshower converter and the calorimeter front face. As a consequence of the increase in the longitudinal development the position in the integral distributions $F$ will be shifted for $\theta \neq 0^{0}$ (figure 12a). Shower fluctuations are also expected to slightly increase due to the additional uncertainty on the starting point of the shower. However this latter effect is small and will be neglected in the following.

The correct shower profile functions for unconverted photons can then be obtained by simply replacing $d$ and $h_{d}$ into equations (12) and (13) with the corresponding $d^{\gamma}$ and $h_{d}^{\gamma}$ values defined as:

$$
\left\{\begin{array}{l}
d^{\gamma}=d+h_{\gamma}\left(\theta_{d}, E=E_{0}\right) \\
h_{d}^{\gamma}\left(\theta_{d}, E=E_{0}\right)=h_{d}\left(\theta_{d}, E=E_{0}\right)+h_{\gamma}\left(\theta_{d}, E=E_{0}\right) \\
h_{\gamma}\left(\theta_{d}, E=E_{0}\right)=\Delta_{\gamma} \sin \left(k_{\gamma} \theta_{d}\right)
\end{array}\right.
$$

where $\Delta_{\gamma}$ and $k_{\gamma}$ are parameters describing the systematic shift. The fitted function $h_{\gamma}\left(\theta_{y}, E_{0}\right)$ is shown in figure $12 \mathrm{~b}$ for $E_{0}=5 \mathrm{GeV}$.

The presence of the preshower converter $\left(1.6 X_{0}\right)$ reduces the total energy deposited in the calorimeter by the converted showers, but does not modify their cluster shape (figure 12a). The parameterization is calculated in the real experimental conditions and only a correction on the final clusterized energy is needed to take into account the energy loss within the lead plate [4]. The profile shapes are also found to be insensitive to the $0.4 T$ magnetic field.

\section{Cluster reconstruction}

The above parameterization of the integral response can predict both the average energy fraction deposited in a single cell of the cluster and the corresponding fluctuations (rms) from the given kinematical conditions. It is then possible to test the hypothesis for the incoming particle to be either an electron or a photon or, alternatively, to resolve close overlapping particles on the basis of their cluster shape. To this purpose a $\chi^{2}$-based fitting procedure is adopted in $X Y$ two-dimensional space after building a $3 \times 3$ cell matrix (nonet) around the local maximum.

The shower description in terms of projected shapes, in spite of the approximation introduced using the factorization (see figure $5 \mathrm{~b}$ ), is rather flexible since the cluster projections 


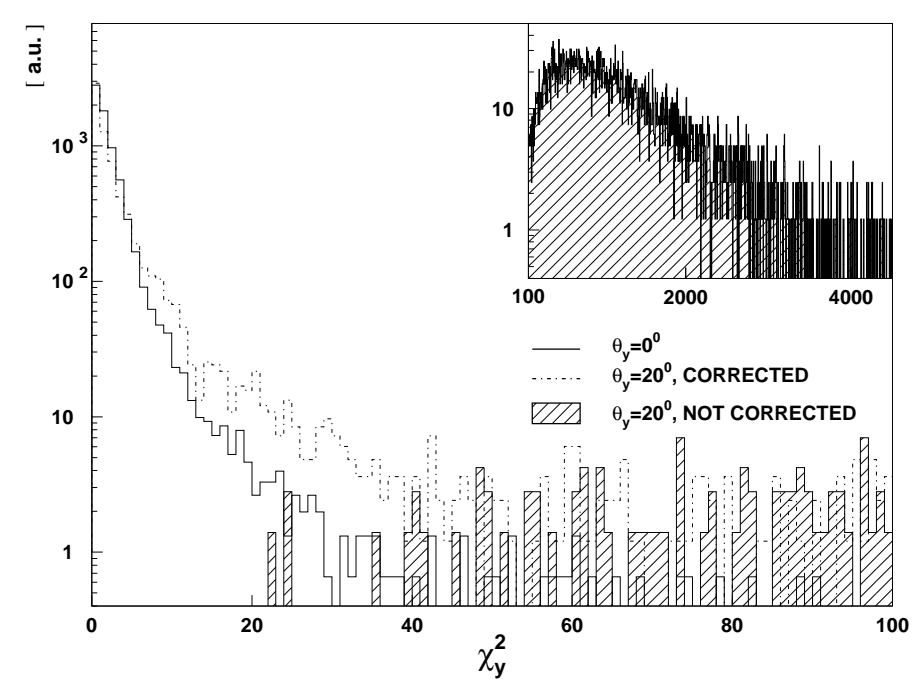

Figure 13: Effect of the described angular correction on $\chi_{y}^{2}$ calculation for $F_{y}\left(y, \theta_{y}=20^{0}, E_{0}=5 \mathrm{GeV}\right)$, compared to the corresponding distribution for $\theta_{y}=0^{0}$.

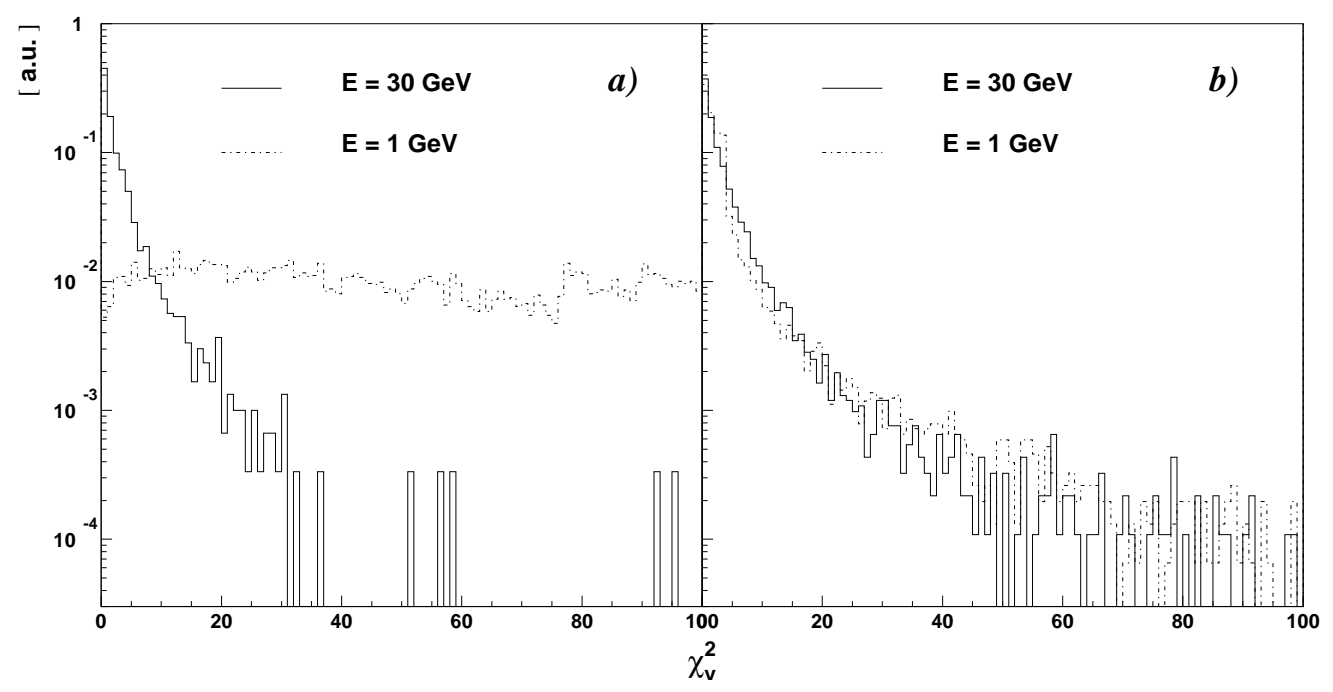

Figure 14: Energy corrections in $\chi_{y}^{2}$ calculation: a) uncorrected distributions and b) corrections have been taken into account. 
along $X$ (or $Y$ ) can be analyzed independently. In particular this property is useful for complex overlaps such as in the NOMAD experimental conditions, where the cluster shape of electrons can be distorted along $Y$ direction by close bremsstrahlung photons due to the bending magnetic field. A separate $\chi_{x}^{2}$ can then be defined using the energy released in the three columns of the nonet:

$$
\chi_{x}^{2}=\sum_{i=1}^{3} \frac{\left[\sum_{j=1}^{3} E_{i j}^{e x p}-F_{x}\left(x_{i}, \theta_{x}, E\right) \times E_{n}\right]^{2}}{\left[\sigma_{x}\left(x_{i}, \theta_{x}, E\right) \times E_{n}\right]^{2}}
$$

where $E_{i j}^{e x p}$ is the measured energy of the block in the $i$-th column and $j$-th row and $x_{i}$ is the distance between the impact point and the $i$-th column. The energy $E_{n}$ provides the overall normalization factor for the energy deposition predicted by the shower profile parameterization. The corresponding $\chi_{y}^{2}$ is defined in a similar way for the three rows placed at distances $y_{j}$ from the impact point.

In addition, a global $\chi^{2}$ referring to the full shower shape in the $X Y$ plane can be evaluated:

$$
\chi^{2}=\sum_{i=1}^{3} \sum_{j=1}^{3} \frac{\left[E_{i j}^{e x p}-F\left(x_{i}, y_{j}, \theta_{x}, \theta_{y}, E\right) \times E_{n}\right]^{2}}{\left[\sigma\left(x_{i}, y_{j}, \theta_{x}, \theta_{y}, E\right) \times E_{n}\right]^{2}}
$$

with the same notation as in the previous equation. In order to minimize the correlations between $\chi_{x}^{2}$ or $\chi_{y}^{2}$ and $\chi^{2}$, this latter is calculated comparing the energy measured in each cell of the nonet to the corresponding predicted value. Since the error on factorization is neglected in the described model for $F\left(x, y, \theta_{x}, \theta_{y}, E\right)$ (section 4 ), this procedure will introduce in principle a small bias on $\chi^{2}$ values. This effect is partially compensated in the $\sigma\left(x, y, \theta_{x}, \theta_{y}, E\right)$ calculation.

The shower parameterization can predict only the energy sharing between the different cells of a cluster. An appropriate choice of the overall normalization factor $E_{n}$ is then needed to compare such relative ratios to the energy distribution measured in the calorimeter. The value of $E_{n}$ should not be necessarily equivalent to the total energy $E$ used to evaluate energy corrections in the shower profile. This will provide a further parameter for the cluster analysis, to be fixed according with the required physical results. Actually, it is more crucial to fully estimate the particle energy in the evaluation of $E$, while a variable normalization through a $\chi^{2}$ minimization can be adopted for $E_{n}$, using only part - even a single cell - of the nonet. The procedure is particularly relevant in order to unfold overlapping clusters and to extract the predicted cluster energy from the calorimeter.

The statistical significance of the $\chi^{2}$ calculation improves by extending the number of blocks considered for the shower shape analysis. On the other hand in order to minimize overlapping problems it is more convenient to define compact showers, even in the presence of large energy deposits. The nonet size $\left(r \sim 3 R_{M}\right)$ was found to be a good compromise for the cluster reconstruction, providing at the same time the containment of most of the particle energy up to large angles of incidence. The energy distributions for single particle used to extract the parameters of the shower description were then obtained considering the most energetic nonet only. Since the final parameterization consists of analytical functions this fact does not exclude the possibility to extend the evaluation of the shower shape outside the nonet. It is also possible to take into account all the tails of the shower if a precise clusterization is needed. In addition the normalization condition inside the nonet imposed to build the $F_{x}$ and $\sigma_{x}\left(F_{y}\right.$ and $\left.\sigma_{y}\right)$ functions provides correlations between different columns (rows) in $\chi^{2}$ calculation. 

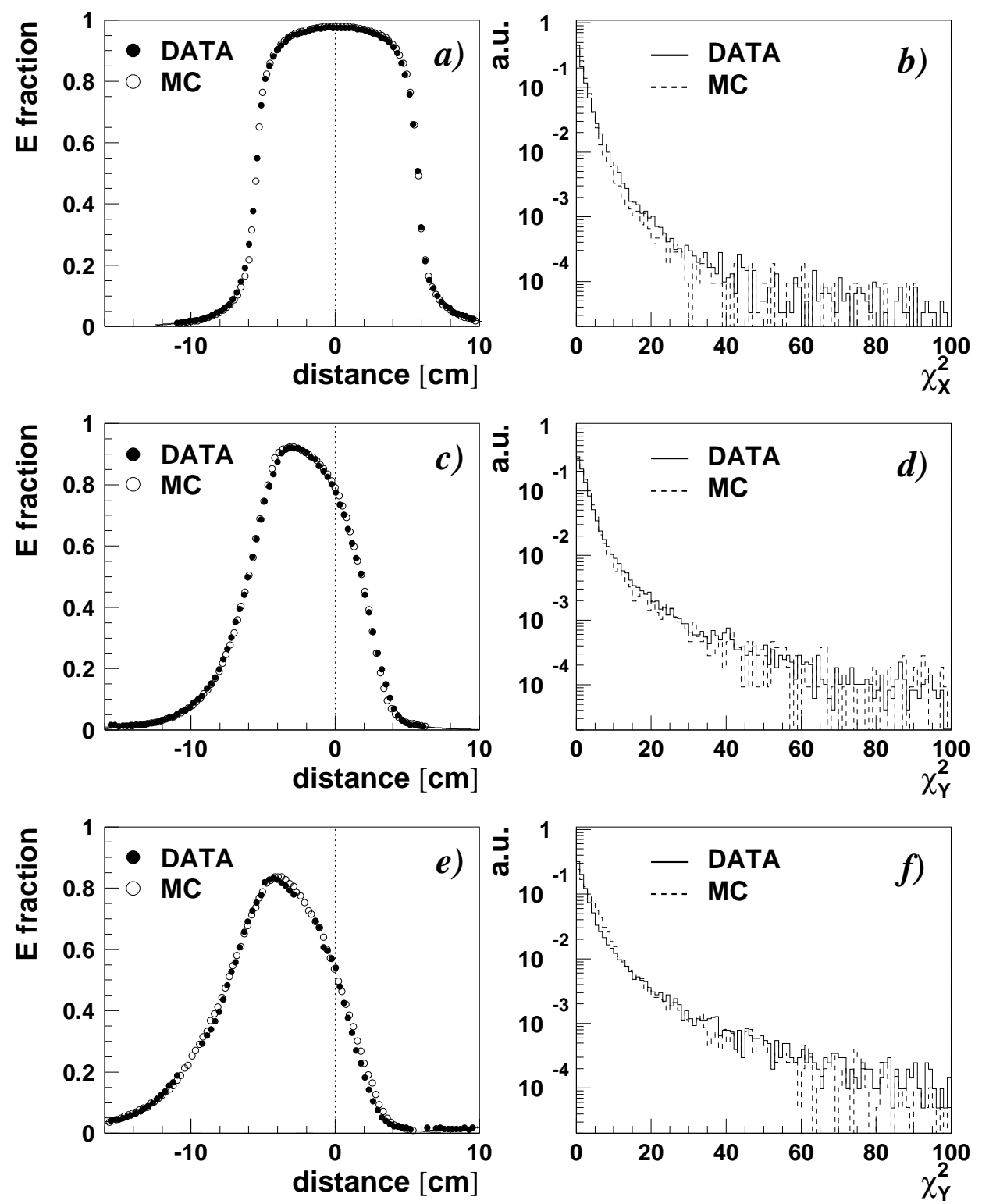

Figure 15: Comparison with test-beam data: a)-b) average behaviour of the integral profile $F_{x}\left(x, \theta_{x}=0^{0}, E=10 \mathrm{GeV}\right)$ and its $\chi_{x}^{2}$ distribution evaluated from parameterization; $\left.\left.c\right)-d\right)$ the same as previous for $F_{y}\left(y, \theta_{y}=10^{0}, E=10 \mathrm{GeV}\right)$; e)-f) $F_{y}\left(y, \theta_{y}=20^{0}, E=5 \mathrm{GeV}\right)$ and the corresponding $\chi_{y}^{2}$ distribution. 
A detailed description of shower fluctuations as a function of the kinematical parameters is introduced for $\chi^{2}$ calculation in order to correctly take into account the containment factor (see section 4.2). On the other hand, due to this effect, the energy distribution observed for a fixed impact point is not exactly gaussian over the full cell size. In particular, large deviations are present in a small region close to the block boundaries. As a consequence the calculated $\chi^{2}$ values (equations (19) and (20)) are not expected to be in perfect agreement with the theoretical $\chi^{2}$ distributions.

\subsection{Parameterization reliability}

The $\chi^{2}$-based algorithm allows a description of the individual electromagnetic showers from the average behaviour predicted by the parameterization. The resulting $\chi^{2}$ distributions can then also give an estimate of the fluctuations associated to the energy deposition. A quantitative test of the described shower model and a full comparison between real testbeam data and MC simulations is thus possible.

Due to the cell size which is of the order of the Molière radius, the integral shower shape is very sensitive to the angle and the energy of the incoming particle. The corrections provided by the parameterization are then needed in order to get an unbiased clusterization procedure. In figures 13 and 14 the effect of such corrections on $\chi^{2}$ distributions is individually shown. In spite of the large variations originally introduced on the shower shape, the final distributions are essentially independent from both angle and energy in the full set of kinematical configurations studied ${ }^{6}$. No significant difference is observed in the $\chi^{2}$ distributions calculated for converted and unconverted photons and electrons.

The shower parameterization can correctly describe MC and real data, as shown in figure 15. In particular, the $\chi^{2}$ distributions (figure 15b-d-f) show a good agreement from the point of view of the shower fluctuations almost independently from the kinematical parameters. This is an indication that longitudinal and lateral shower development are well reproduced in the shower model.

\subsection{Particle identification}

The most natural application of the shower shape calculation is the identification of the electromagnetic showers. The $\chi^{2}$ calculation described above is independent from both angle and energy of the particle and this allows to fix the criteria $\left(\chi^{2}\right.$ cuts) for particle identification on the basis of the required rejection factor only.

A cut on the global $\chi^{2}$ which takes into account the full shower shape in the $X Y$ plane or, alternatively, combined cuts on $\chi_{x}^{2}$ and $\chi_{y}^{2}$ will produce the highest rejection. On the other hand the presence of three different $\chi^{2}$ can reduce overlap problems since the three $\chi^{2}$ cuts can be separately optimized.

In figure 16 the $e / \pi$ separation curves are shown for $E_{0}=5 \mathrm{GeV}$ and $\theta_{x}=\theta_{y}=10^{\circ}$. For a 0.90 efficiency in the electron identification a residual $\pi$ contamination less than 0.02 can be achieved on single-particle clusters with a global $\chi^{2}$ cut ${ }^{7}$.

\footnotetext{
${ }^{6}$ The $\chi^{2}$ distributions before corrections do not show long tails for high energy particles (figure 14a) because of an overestimation of the $\sigma$ functions which are energy-dependent (equation (16)). This would result in an increase of the pion contamination.

${ }^{7}$ This is a conservative result since the global $\chi^{2}$ is affected by the errors on $X Y$ factorization and correlations are neglected in the global $\sigma$ evaluation. It can be improved by considering for instance a linear
} 


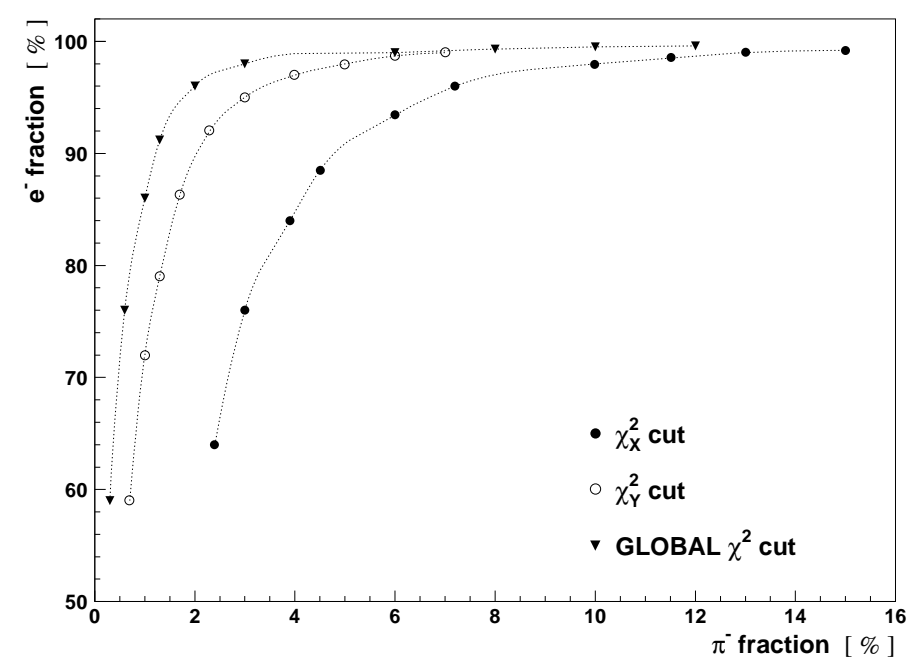

Figure 16: Efficiency for e/ $\pi$ separation as a function of the $\chi^{2}$ cut at $\theta_{x}=10^{0}, \theta_{y}=10^{0}$ and $E_{0}=5 \mathrm{GeV}$. The statistical errors are negligible.

The results for $e / \pi$ separation are almost independent from the impact angle. The rejection efficiency is expected to decrease by reducing the impact energy. This reduction for low energies is due on one side to the larger shower fluctuations of electromagnetic showers and on the other to the fact that hadronic and electromagnetic showers are closer to each other.

\subsection{Coordinate measurement}

From the parameterization of the shower shape the momenta of the integral energy distribution can be calculated as a function of the kinematical parameters $\theta_{x}, \theta_{y}$ and $E$ :

$$
M_{d}^{n}\left(d, \theta_{d}, E\right)=\sum_{k=-1}^{1} F_{d}\left(d+k \Delta d_{0}, \theta_{d}, E\right) \times\left[d+k \Delta d_{0}\right]^{n} \quad d=x, y
$$

where the sum extends over the three rows (columns) of the nonet. In the above expression only a nonet is considered for simplicity.

Due to the hodoscopic structure of the calorimeter, the reconstructed centre of gravity position inside the nonet $\left(M_{x}^{1}, M_{y}^{1}\right)$ is related to the true position by a non-linear relationship [8]. On the other hand the strong dependence from the impact angle and energy of the particle can introduce a large bias in the estimation of the position through the energy deposition measured in the calorimeter. In particular, the systematic shift $h_{d}\left(\theta_{d}, E\right)$ is responsible for the offset observed in the reconstructed position, which increases with the impact angle (figure 17a).

combination $\bar{\chi}^{2}=\left(\chi_{x}^{2}+w \chi_{y}^{2}\right) /(1+w)$ where $w$ is a fixed weight. On the other hand such parameter should be optimized according to the physical analysis. 

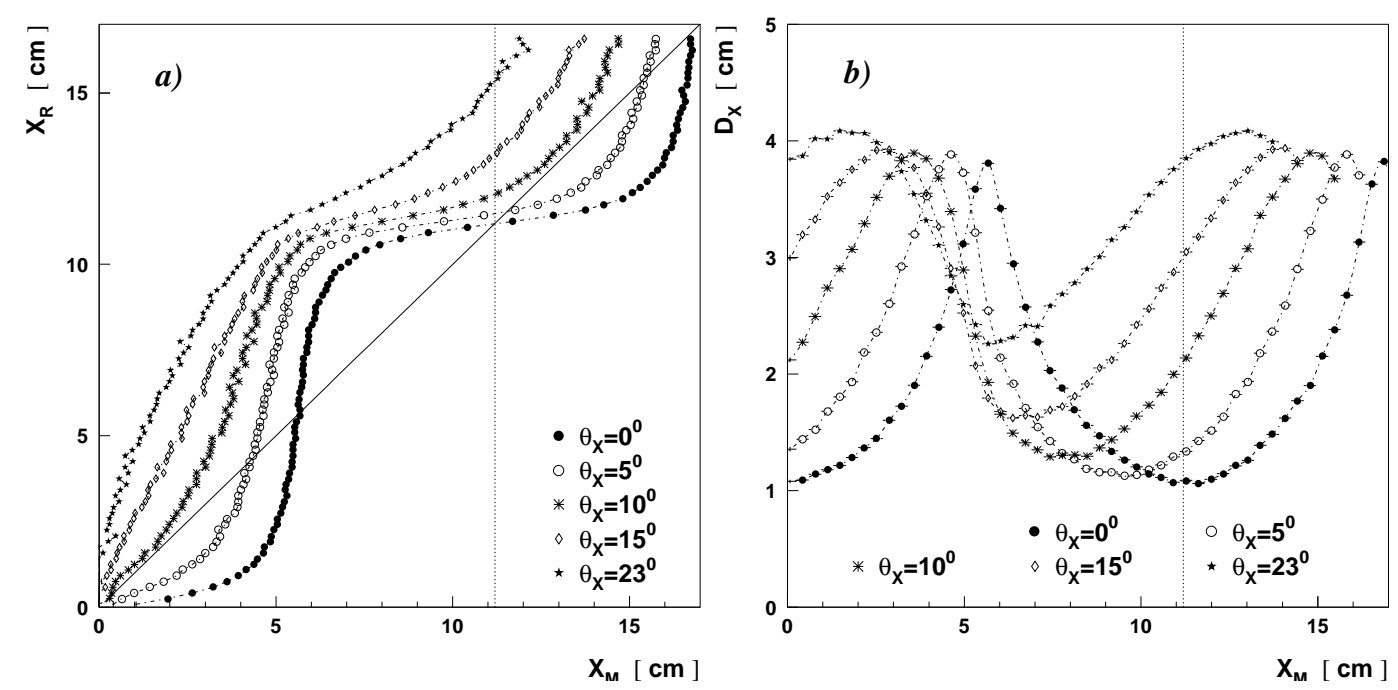

Figure 17: Coordinate reconstruction for $e^{-}$of $E=5 \mathrm{GeV}$ at different $\theta_{x}$ : a) reconstructed (centre of gravity) $M_{x}^{1}$ position and b) cluster radius $D_{x}^{2}$ as a function of the impact point. The vertical lines correspond to the block centre.
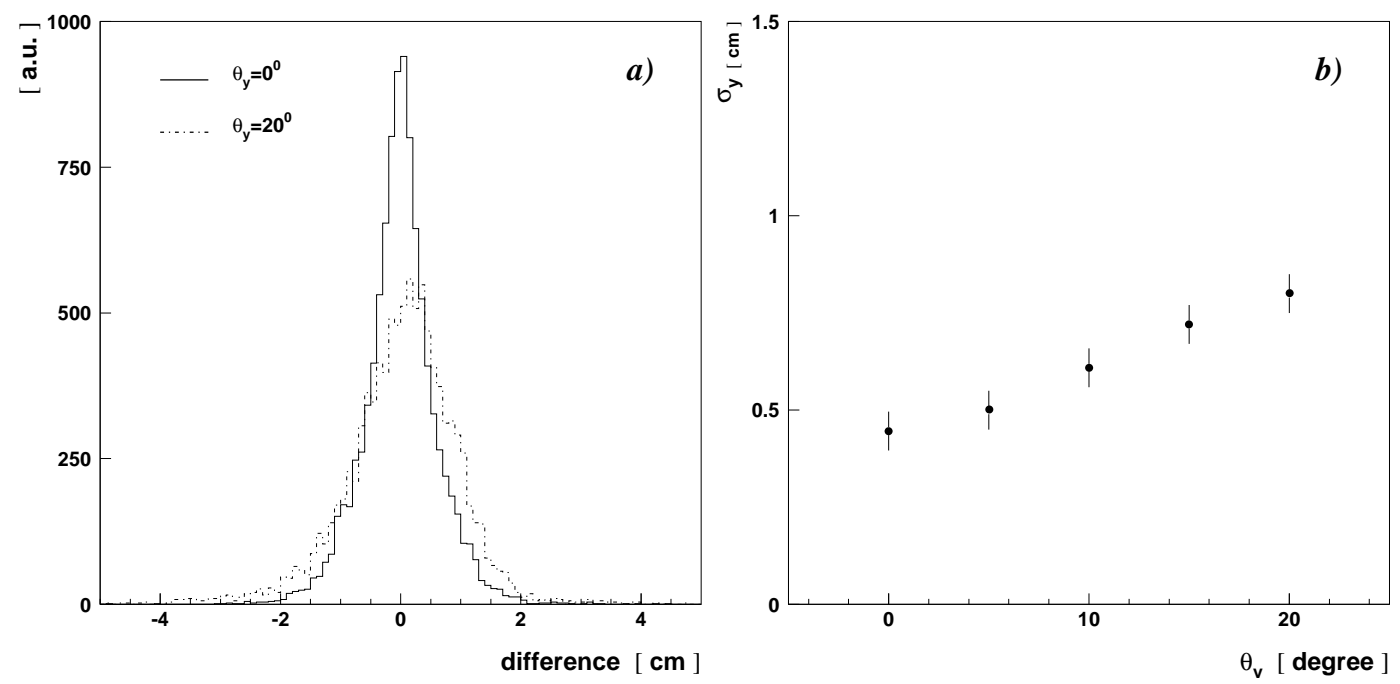

Figure 18: Coordinate reconstruction obtained from the shower parameterization for $\gamma$ at $E=5 \mathrm{GeV}:$ a) difference between the real and reconstructed $Y$ position at different $\theta_{y}$ and b) overall space resolution as a function of the angle $\theta_{y}$. 
A similar behaviour can be seen in the momenta evaluated with respect to the centre of gravity $M_{d}^{1}$ :

$$
\begin{aligned}
D_{d}^{n}\left(d, \theta_{d}, E\right) & =\sum_{k=-1}^{1} F_{d}\left(d+k \Delta d_{0}, \theta_{d}, E\right) \times \\
& \times\left[\left(d+k \Delta d_{0}\right)-M_{d}^{1}\left(d, \theta_{d}, E\right)\right]^{n} \quad d=x, y
\end{aligned}
$$

with the same notation as in the previous equation.

On the average the cluster radius $D_{d}^{2}$ is larger and also less position dependent for $\theta \neq 0^{0}$ then $\theta=0^{0}$ (figure 17b) since in the former case the shower extends radially on several blocks.

The expressions (21) and (22) can predict the true impact position directly from the shower parameterization. The advantage is that angular and energy effects are already taken into account in the shape calculation. The results obtained for a particular angle $\theta_{d}^{0}$ are equivalent to the direct fit to the non-linear relationship between the reconstructed centre of gravity and the true position [4].

An alternative procedure uses a $\chi^{2}$ minimization as a function of the estimated impact point. This algorithm can be applied within the tracking errors for electrons or within the full cell size for photons. It is more powerful then the previous procedure since it relates the calculated position not only to the average profile, but also to the expected shower fluctuations. On the other hand the computation time required is longer than in the previous case where only analytical functions are evaluated and the method is in principle sensitive to the starting point of the fitting procedure.

The distributions of the difference between the true $Y$ position and the one reconstructed from $M_{y}^{1}$ are reported in figure 18a for converted photons. An overall space resolution $\sigma_{y} \sim$ $0.4 \mathrm{~cm}$ can be obtained at $\theta=0^{0}$ for particles randomly distributed over the full cell size. Actually the space resolution is a function of the impact coordinate on the cell front face and a variation as high as a factor of three can be found between the centre and the boundaries of the block [4]. Since for $\theta \neq 0^{0}$ the additional contributions coming from the longitudinal component of the shower increase the fluctuations associated to the integral radial profile (equation (16)), the overall space resolution is also expected to be sligthly worse as a function of the impact angle. The effect is shown in figure $18 \mathrm{~b}$.

\subsection{Energy reconstruction}

The prediction of the energy deposited in the cells of the cluster and of the corresponding fluctuations allows to extract from the calorimeter the full energy associated to the incoming particle. This is important in order to resolve close overlaps and to build neutral clusters. The procedure is partially independent from a pure $\chi^{2}$ evaluation as defined in section 5 , since for hard overlaps the $\chi^{2}$ 's calculated on the full nonet are higher. On the other hand it is possible to clusterize the energy according to the shower profile predictions in the electromagnetic hypotesis, regardeless of the $\chi^{2}$ values previously found.

The energy of the particle can be estimated from the comparison between the predicted energy and the one measured in the calorimeter. The errors are computed by summing in quadrature the shower fluctuations $\sigma_{i j}^{t h}$ and the electronic noise from pedestal fluctuations $\sigma_{\text {ped }}$. 


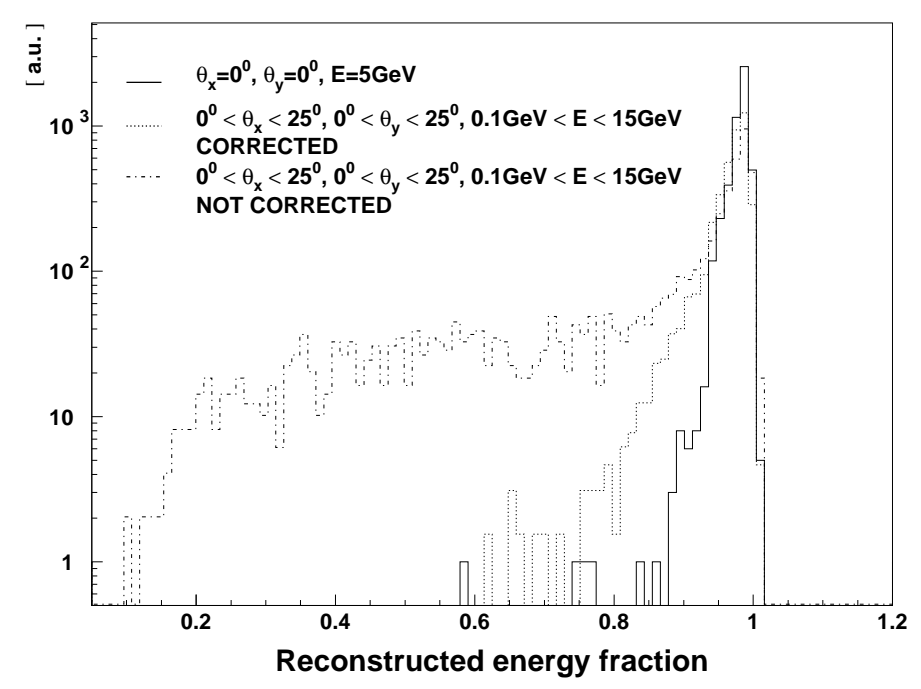

Figure 19: Effect of the angular and energy corrections in shower shape parameterization on the $e^{-}$reconstructed energy.

The reconstructed energy fraction with respect to the original Monte Carlo energy is shown in figure 19 for single particle clusters. It is clear from the displayed distributions that the above procedure cannot be applied without taking into account angular and energy corrections in the shower profile calculation since this would result in a significant energy loss. On the other hand the measured energy deposition is well described by the shower model for a large set of kinematical configurations. The overall energy resolution obtained is $0.016\left(0.5<E<15 \mathrm{GeV}, 0^{0}<\theta_{x}, \theta_{y}<25^{0}\right)$.

\section{Application to neutrino events}

As already discussed in section 1, the NOMAD experiment strongly relies on a good reconstruction of the kinematical variables (momenta and energies) and on the identification of the charged particles produced in neutrino interactions. This is particularly relevant for the $e^{-} \bar{\nu}_{e} \nu_{\tau}$ channel where, due to the bremsstrahlung radiation in the magnetic field, a calorimetric measurement is required to improve the momentum resolution of the fitted electron track. On the other hand, the kinematical criteria applied for the $\tau$ search require isolation conditions on the leading electron. The shower shape fitting procedure described in the previous section can provide at the same time a powerful tool in helping electron identification against pions and a way to study electron isolation conditions since it is very sensitive to close overlaps. To this purpose, the factorization of the shower shapes in $X$ and $Y$ projections is particularly relevant, since the bremsstrahlung radiation associated to the electron in the magnetic field can produce a stream of close $\gamma$ clusters only along the bending $(Y)$ direction. Moreover this is a purely calorimetric technique, almost decoupled from the track fitting procedure.

In order ot test the previously defined algorithm for real neutrino interactions, a sample of $\nu_{e}$ induced Charged Current events $\left(\nu_{e} C C\right)$ was fully simulated into the NOMAD apparatus using the GEANT package [5]. Figure 20a-b shows the $\chi_{x}^{2}$ and $\chi_{y}^{2}$ distributions respectively 


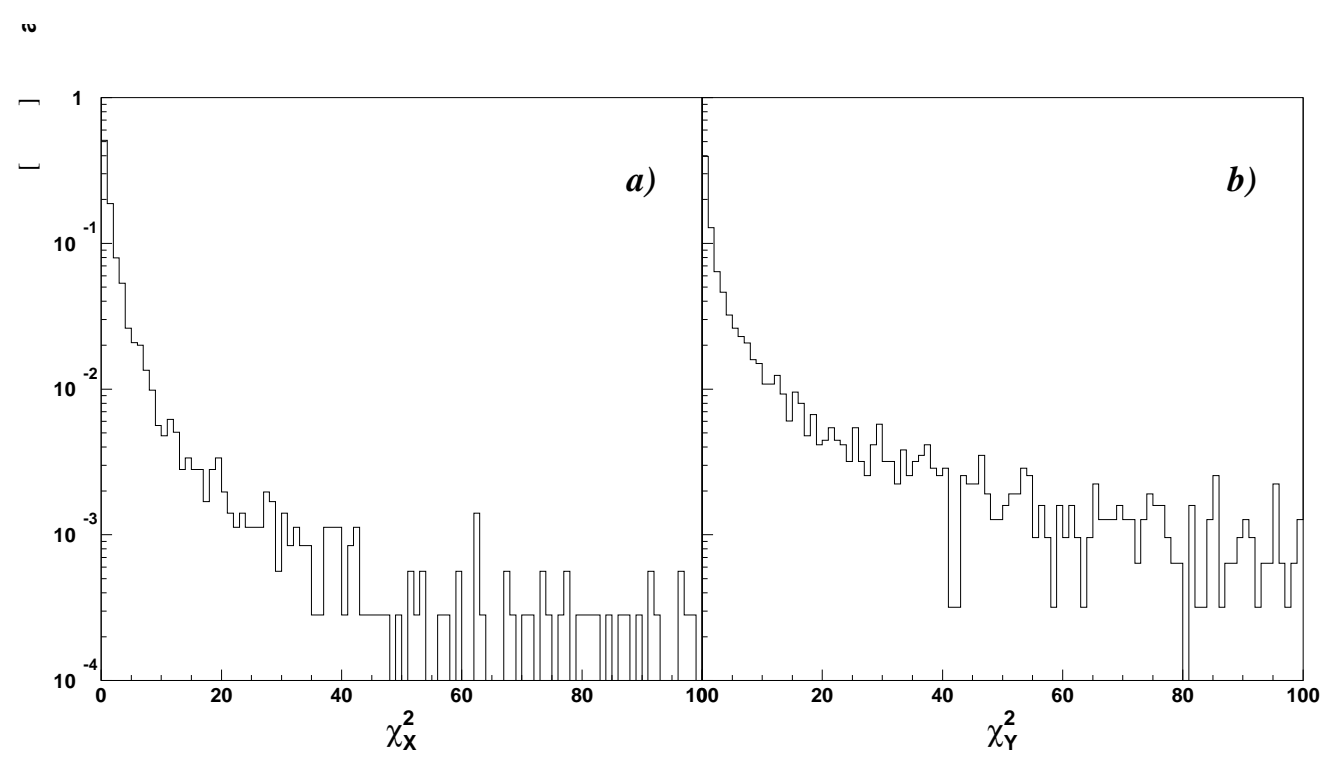

Figure 20: a) $\chi_{x}^{2}$ and b) $\chi_{y}^{2}$ distributions of a sample of prompt electrons in $\nu_{e} C C$ simulated events. The distributions are integrated over all the electron energies and all the incidence angles on the front face of the electromagnetic calorimeter.

for prompt electrons in $\nu_{e} C C$ events. While the $\chi_{x}^{2}$ distribution is in good agreement with those calculated for test-beam electrons (see figure 15), the $\chi_{y}^{2}$ distribution has a broader shape due to the presence of bremsstrahlung photons overlapping the electron cluster along the magnetic field direction. The corresponding efficiency for $e / \pi$ separation as a function of a $\chi_{x}^{2}$ cut is presented in figure 21 for an electron energy $E>2 \mathrm{GeV}$. In spite of the good agreement observed for the $\chi_{x}^{2}$ distributions between single particle (data and MonteCarlo) and $\nu_{e} C C$ simulated events, figure 21 shows a deterioration of the $e / \pi$ separation. This effect is due both to the presence of low energy electrons with wide angular distribution and to the lower average energy of the hadrons produced in jet fragmentation, with respect to the prompt electron component.

The correct measurement of the electron energy released in the electromagnetic calorimeter is an additional requirement which is very important to determine the neutral component of the transverse momentum. This implies knowledge of the electromagnetic shower shapes to clusterize both the electron and the photons emitted by bremmstrahlung radiation. Since overlaps strongly affect the energy calculation, a recursive procedure based on a $\chi^{2}$ minimization with respect to the impact point, is applied as discussed in section 5 . In figure 22 the resolution of the electron cluster built according to the shower profile predictions is shown for prompt electrons having $E>2 \mathrm{GeV}$. In spite of the large variation of the kinematical parameters $\left(E, \theta_{x}, \theta_{y}\right)$ of the incident electrons most of the shower energy is properly reconstructed, with an overall energy resolution of $\Delta E / E \sim 0.015(E>2 \mathrm{GeV})$.

\section{Conclusions}

A method to parameterize the integral electromagnetic shower shape in the NOMAD leadglass calorimeter as a function of the angle and energy of the impact particle was discussed for $0.1<E<80 \mathrm{GeV}$ and $0^{0}<\theta<25^{0}$. Both the average shower profiles and the 


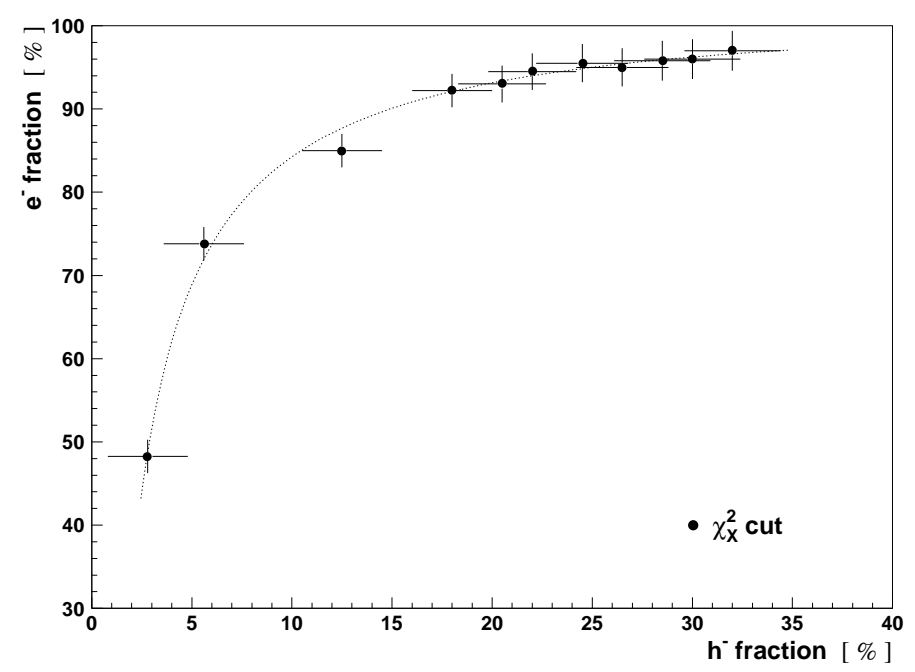

Figure 21: Efficiency for e/ $\pi$ separation as a function of $\chi_{x}^{2}$ for primary charged tracks with $E>2 \mathrm{GeV}$ in a sample of $\nu_{e} C C$ simulated events. Only statistical errors are shown.

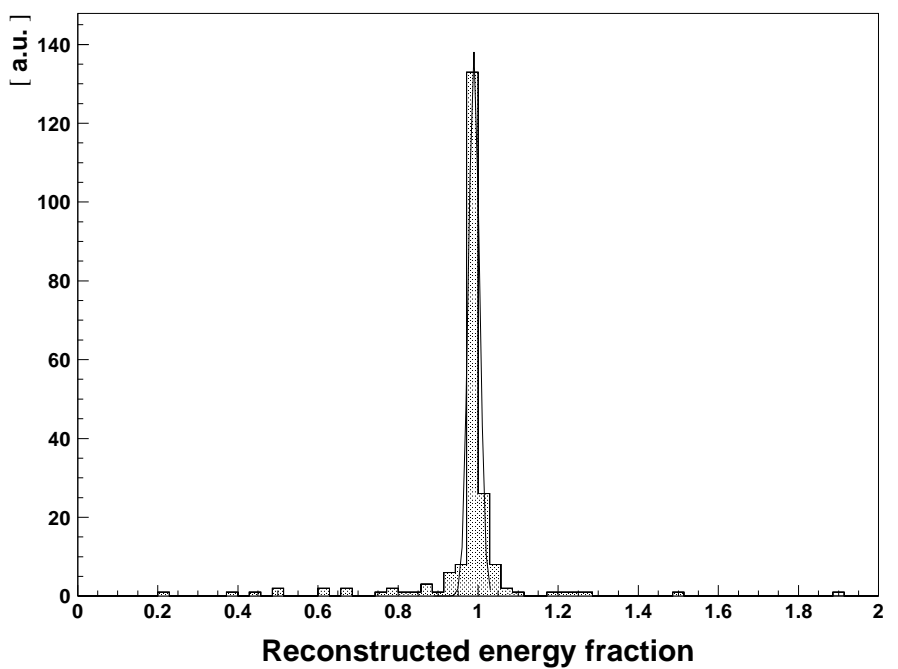

Figure 22: Resolution of the cluster built according to the shower profile calculation for prompt electrons in $\nu_{e} C C$ events. The extracted cluster is compared with the Monte Carlo cluster associated to the charged track reaching the calorimeter. 
fluctuations were studied for electrons and photons. The developed model was applied to particle identification through a $\chi^{2}$ based fitting procedure. The algorithm allows one to obtain a residual $\pi$ contamination around 0.02 for an electron identification efficiency of 0.9 in the case of isolated clusters at $E=5 \mathrm{GeV}$. The shower parameterization was also used to reconstruct the coordinates and energy of the impact particles at the calorimeter front face. An overall space resolution of $0.6 \mathrm{~cm}$ (at $\theta=10^{\circ}$ and $E=5 \mathrm{GeV}$ ) was obtained for converted photons. The corresponding overall energy resolution was $\Delta E / E \sim 0.016$ $\left(0.5<E<15 \mathrm{GeV}, 0^{0}<\theta<25^{0}\right)$. The described algorithms were eventually applied to a simulated sample of $\nu_{e} C C$ events, in the real NOMAD experimental conditions. The results are in good agreement with those expected from test beam data when taking into account the bremsstrahlung radiation of real electrons in the presence of the NOMAD magnetic field.

\section{Acknowledgements}

We would like to thank D. Sillou for usefull discussions in the early stage of the work. Special thanks to the technical staff of the CERN, Cosenza, Firenze, Moscow, Padova, Pavia and Pisa groups for their invaluable contributions. The financial support of the INFN and INR is gratefully acknowledged. Finally, best thanks to L. DiLella and L. Camilleri for their continuous help and lively discussions.

\section{References}

[1] NOMAD proposal and addenda:

CERN-SPSLC/91-21, CERN-SPSLC/91-48, CERN-SPSLC/91-53, CERN-SPSLC/93-19, CERN-SPSLC/94-21, CERN-SPSLC/94-28.

[2] NOMAD Collaboration, Nucl. Instr. and Meth. A404 (1998) 96.

[3] D. Autiero et al. Nucl. Instr. and Meth. A373 (1996) 358.

[4] D. Autiero et al. Nucl. Instr. and Meth. A387 (1997) 352.

[5] CERN Program Library Long Writeup W5013, edition March 1994.

[6] W.R. Nelson et al. Phys. Rev. 149 (1966) 210.

[7] G. Bathow et al. Nucl. Phys. B20 (1970) 592.

[8] G.A. Akopdjanov et al. Nucl. Instr. and Meth. 140 (1977) 441.

[9] E. Longo and I. Sestili Nucl. Instr. and Meth. 128 (1975) 283.

[10] B. Rossi High Energy Particles, Prentice-Hall 1952.

[11] A. Lednev Nucl. Instr. and Meth. A366 (1995) 292. 